\title{
A Covariant model for the nucleon and the $\Delta$
}

\author{
G. Ramalho ${ }^{1,2}$, M.T. Peña ${ }^{2,3}$ and Franz Gross ${ }^{1,4}$ \\ ${ }^{1}$ Thomas Jefferson National Accelerator Facility, Newport News, VA 23606 \\ ${ }^{2}$ Centro de Física Teórica de Partículas, Av. Rovisco Pais, 1049-001 Lisboa, Portugal \\ ${ }^{3}$ Department of Physics, Instituto Superior Técnico, \\ Av. Rovisco Pais, 1049-001 Lisboa, Portugal and \\ ${ }^{4}$ College of William and Mary, Williamsburg, Virginia 23185
}

(Dated: October 30, 2018)

\begin{abstract}
The covariant spectator formalism is used to model the nucleon and the $\Delta(1232)$ as a system of three constituent quarks with their own electromagnetic structure. The definition of the "fixed-axis" polarization states for the diquark emitted from the initial state vertex and absorbed into the final state vertex is discussed. The helicity sum over those states is evaluated and seen to be covariant. Using this approach, all four electromagnetic form factors of the nucleon, together with the magnetic form factor, $G_{M}^{*}$, for the $\gamma N \rightarrow \Delta$ transition, can be described using manifestly covariant nucleon and $\Delta$ wave functions with zero orbital angular momentum $L$, but a successful description of $G_{M}^{*}$ near $Q^{2}=0$ requires the addition of a pion cloud term not included in the class of valence quark models considered here. We also show that the pure $S$-wave model gives electric, $G_{E}^{*}$, and coulomb, $G_{C}^{*}$, transition form factors that are identically zero, showing that these form factors are sensitive to wave function components with $L>0$.
\end{abstract}

\section{INTRODUCTION}

Experimentally the main source of information on the internal structure of baryons lies in the electro- and photo-excitation of the nucleon, and is parametrized in terms of electromagnetic form factors. The precise elastic electron-proton polarization transfer experiments undertaken at Jlab [1, 2, 3] disclosed results at odds with previous data, triggering an intense discussion about the shape of the nucleon [4, 5] (a review of the subject can be found in Refs. [6, 7]). Recent measurements of the $\gamma N \rightarrow \Delta$ transition raise further questions. How much of this process is due to the three valence quarks in the $\Delta$, and how much to the meson-nucleon, or "pion cloud" contribution? Experimental progress has been impressive, with the recent accumulation of high precision data over a wide momentum transfer $\left(Q^{2}\right)$ range. Just ten years ago, for example, the sign of the electric and magnetic ratio for the $\gamma N \rightarrow \Delta$ transition, was not even known beyond the photon point $\left(Q^{2}=0\right)$. New precise data have been collected from MAMI [8], LEGS [9], MIT-Bates [10] and Jlab [11, 12] in a region $Q^{2} \leq 6 \mathrm{GeV}^{2}$ $\left(q^{2}=-Q^{2}\right.$ is the squared transferred momentum).

This is the second in a series of papers using the covariant spectator theory to study the implications of modeling the baryon resonances as covariant bound states of three valence constituent quarks (CQ). In this model the structure of the $\mathrm{CQ}$ (including an anomalous magnetic moment) arises from the dressed $\gamma \rightarrow q \bar{q}$ interactions which give rise to the familiar meson structure of the vector dominance model, and these contributions are not included in the wave function of the nucleon. This language differs significantly from the light-cone formalism, where all of this vector dominance physics must be included in higher, non-valence components of the Fock- space expansion of the nucleon wave function (for further discussion, see [5]). In our first paper [5] (referred to as Ref. I) we showed that a very simple pure $S$-wave model of the nucleon, based on a covariant generalization of a simple non-relativistic $S U(2) \times S U(2)$ wave function for three valence $\mathrm{CQ}$, could describe the four elastic nucleon form-factors very well. Our best model used 8 parameters: two to model the "radial" structure of the nucleon wave function, two to describe the anomalous magnetic moments of the up and down quarks, one to allow for an overall renormalization of the quark charge at very large $Q^{2}$, and three to describe the vector dominance structure of the four quark form factors. Three of these parameters were fixed, leaving 5 to be varied during the fit.

The present paper extends this model to the description of the $\gamma N \rightarrow \Delta$ transition. As required by the model we use the same CQ form factors and nucleon wave function as fixed in Ref. I. The only freedom remaining is the structure of the $\Delta$ wave function, and in this paper we study the consequences of the assumption that this wave function is a pure symmetric $S$-wave system of three valence $\mathrm{CQ}$ with total spin and isospin equal to $3 / 2$ (described with two parameters). We conclude that although the electromagnetic form factors of the nucleon alone may be described with such a simple ansatz based on orbital $S$-waves only, the simultaneous description of all the $\gamma N \rightarrow \Delta$ multipole transition form factors demands partial waves with $L>0$. This is in agreement with the findings of the first non-relativistic quark models which yield a magnetic dipole (M1) form factor but no contributions to the electric (E2) and Coulomb (C2) quadrupole form factors [13], since they did not include single quark $D$-states, either in the nucleon or in the $\Delta$ wave function.

In Section II we introduce the $\Delta$ wave function, after 




FIG. 1: Baryon-quark wave function defined in Eq. (2.1).

a short redefinition of the nucleon wave function. In Section III the issues related to the basis for the polarization states are briefly reviewed. In Section IV the current corresponding to the $\mathrm{N} \rightarrow \Delta$ electromagnetic transition is introduced, and in Section $\mathrm{V}$ the form factors are calculated. Section VI presents the results. Section VII summarizes the work and draws conclusions.

\section{RELATIVISTIC $S$-WAVE FUNCTIONS FOR BARYONS}

In the spectator framework $[5,14,15,16,17,18,19$, $20,21,22,23]$, a general baryon with four-momentum $P$ and mass $M$ is described by a wave function for an off-shell quark and an on-mass-shell diquark-like cluster, defined by

$$
\Psi(P, k)=\left(m_{q}-\not p_{1}\right)^{-1}\langle k|\Gamma| P\rangle,
$$

where $\Gamma$ is the vertex function describing the coupling of an incoming on-shell baryon with mass $M$ to an outgoing off-shell quark and an on-shell quark pair (the $d i$ quark). The quark pair is non-interacting with a continuous mass distribution varying from $2 m_{q}$ to infinity. All of the matrix elements in the spectator theory involve an integral over this mass distribution, and for simplicity this integral is approximated by fixing this mass at a mean value, $m_{s}$ (a parameter of the theory that scales out of the form factors, but can be fixed by deep inelastic scattering, as described in Ref. I). The diquark four-momentum, $k=P-p_{1}$, is constrained by its onmass-shell condition $k^{2}=m_{s}^{2}$. The quark has dressed mass $m_{q}$ and four-momentum $p_{1}$. This wave function is shown diagrammatically in Fig. 1 .

Following Refs. I and [14], we write the baryon states in terms of the quark spin and "fixed-axis" diquark polarization states, labeled by $\varepsilon_{p}^{\mu}$. These vectors were discussed in detail in Ref. I and [14]. We use them, instead of the diquark helicity vectors, since the latter depend not only on the magnitude of the diquark momentum, but also on its direction, while here we want to consider $S$-state orbital effects alone. Considering the "fixed-axis" polarization states we have shown [5, 14] that the wave function for the nucleon transforms as a Dirac spinor under a Lorentz operation. This implies that the model is covariant and assures the covariance of the electromagnetic current elements. The method was tested for the nucleon alone in Ref. I; here we test it for the $\Delta$.

\section{A. $S$-wave nucleon wave function}

The manifestly covariant $S$-wave nucleon (with mass $m$ ) wave function was introduced in Ref. I

$$
\begin{aligned}
\Psi_{N \lambda_{n}}(P, k) & =\frac{1}{\sqrt{2}} \psi_{N}(P, k) \phi_{I}^{0} u\left(P, \lambda_{n}\right) \\
& -\frac{1}{\sqrt{6}} \psi_{N}(P, k) \phi_{I}^{1} \gamma_{5} \notin_{P}^{*} u\left(P, \lambda_{n}\right) .
\end{aligned}
$$

Here $u\left(P, \lambda_{n}\right)$ is a four-component Dirac spinor, and $\psi_{N}$ is a scalar function that specifies the relative shape of both spin-isospin $(0,0)$ and spin-isospin $(1,1)$ diquark components. In the following we will sometimes simplify the notation by suppressing reference to the polarization $\lambda_{n}$ of the nucleon, and write the nucleon spinor as $u\left(P, \lambda_{n}\right) \rightarrow u(P)$.

In Eq. (2.2), $\phi_{I}$ gives the isospin states of the quarkdiquark system $(I= \pm 1 / 2$ is the isospin projection of the nucleon)

$$
\begin{aligned}
& \phi_{I}^{0}=\xi^{0 *} \chi^{I} \\
& \phi_{I}^{1}=-\frac{1}{\sqrt{3}} \tau \cdot \xi^{1 *} \chi^{I},
\end{aligned}
$$

where $\xi^{i}(i=0,1)$ represents the two diquark isospin states, and

$$
\chi^{+\frac{1}{2}}=\left(\begin{array}{l}
1 \\
0
\end{array}\right)=n \quad \chi^{-\frac{1}{2}}=\left(\begin{array}{l}
0 \\
1
\end{array}\right)=p .
$$

More details can be found in Ref. I.

The spin-1 component of the wave function depends on the fixed-axis diquark polarization vectors $\varepsilon_{\lambda P}=$ $\left(\varepsilon_{0}, \varepsilon_{x}, \varepsilon_{y}, \varepsilon_{z}\right)$, with $\lambda=0, \pm 1$ the polarization index. The explicit expressions for $\varepsilon_{\lambda P}$ are given in the next Section (Eqs. (3.2) for $m_{H}=m$ ). Here we emphasize that the polarization vectors are written in terms of the nucleon momentum $P$, instead of the diquark momentum $k$. This dependence gives $\Psi_{N}$ the correct non relativistic limit and also assures that $\Psi_{N}$ satisfies the Dirac equation [5].

Note that (2.2) is written in terms of $\varepsilon_{P}^{*}$, allowing the interpretation of $\Psi_{N}$ as an amplitude for an incoming nucleon and an outgoing diquark in the final state (see Fig. (1).

In this paper we only consider transitions from the nucleon to the $\Delta$ in which the diquark remains a spectator, with its spin and isospin unchanged during the transition. Since the diquark in the $\Delta$ must have spin-isospin quantum numbers $(1,1)$ [as discussed below], only the spinisospin $(1,1)$ component of the nucleon wave function is needed. It is convenient to introduce a new notation for this component, and rewrite the $(1,1)$ component of Eq. (2.2) as

$$
\begin{aligned}
\Psi_{N \lambda_{N}}(P, k) & \rightarrow \Psi_{N \lambda_{N}}^{(1,1)}(P, k) \\
& =-\frac{1}{\sqrt{2}} \psi_{N}(P, k) \phi_{I}^{1} \varepsilon_{P}^{\alpha *} U_{\alpha}\left(P, \lambda_{N}\right)
\end{aligned}
$$


where $\alpha$ is a vector index, and

$$
U_{\alpha}\left(P, \lambda_{N}\right) \equiv \frac{1}{\sqrt{3}} \gamma_{5}\left(\gamma_{\alpha}-\frac{P_{\alpha}}{m}\right) u\left(P, \lambda_{N}\right) .
$$

Because $\varepsilon_{P} \cdot P=0$, this definition is equivalent to the one presented in [5]. However, the spinor (2.6) has the properties

$$
\begin{aligned}
P^{\alpha} U_{\alpha}\left(P, \lambda_{N}\right) & =0 \\
(m-P) U_{\alpha}\left(P, \lambda_{N}\right) & =0
\end{aligned}
$$

which are very convenient for the actual calculation of the matrix elements of the electromagnetic current. Note also that

$$
(m-P) \Psi_{N \lambda_{N}}(P, k)=0 .
$$

The part of the wave function that depends on the magnitude of the relative momentum is modeled by the two parameter function used in Ref. I:

$$
\psi_{N}(P, k)=\frac{N_{0}}{m_{s}\left(\beta_{1}+\chi_{N}\right)\left(\beta_{2}+\chi_{N}\right)} .
$$

Here $N_{0}$ is a normalization constant and $\beta_{i}$ with $i=$ 1,2 are range parameters in units of $m m_{s}$. All of these parameters were fixed in Ref. I, and we use the same values in this paper. The dimensionless variable $\chi_{N}$ is defined as

$$
\chi_{N}=\frac{\left(m-m_{s}\right)^{2}-(P-k)^{2}}{m m_{s}} .
$$

Since the baryon and the diquark are both on-shell, the wave function (2.9) can depend only on the variable $(P-k)^{2}$ (as required by the Hall-Wightman theorem). In Appendix G] we show that Eq. (2.9) assures the asymptotic behavior for the nucleon form factors $G_{E}$ and $G_{M}$ will be $1 / Q^{4}$ times logarithmic corrections as expected from perturbative QCD (pQCD).

This formalism is not restricted to the $S$-wave case presented here. It can be extended to states with higher orbital angular momentum. This will be the subject of future work.

The wave function (2.5) has a very simple physical interpretation. It is a spin $1 / 2$ state composed of spin 1 and spin $1 / 2$ constituents. This spin content is discussed in detail in Appendix $\mathrm{A}$.

\section{B. $S$-wave $\Delta$ wave function}

The $S$-state wave function for the $\Delta$ (with mass $M$ ) is defined similarly to $(1,1)$ component of the nucleon wave function in Eq. (2.5) above. Non-relativistically a pure $S$-wave spin $3 / 2$ Delta state can be written as a direct product of spin $1 / 2$ quark and a spin-1 diquark. Many details of the nonrelativistic construction of the $\Delta$ wave, and its relativistic generalization, are discussed in Appendix B In this section we summarize the results.

In parallel to Eq. (2.5), the $S$-state Delta covariant wave-function can be written

$$
\Psi_{\Delta \lambda_{\Delta}}(P, k)=-\psi_{\Delta}(P, k) \tilde{\phi}_{I^{\prime}} \varepsilon_{P}^{\beta *} w_{\beta}\left(P, \lambda_{\Delta}\right),
$$

where $\tilde{\phi}_{I^{\prime}}$ is the isospin part of the $\Delta$ wave function (including a diquark with isospin 1 , and playing the same role as the nucleon isospin function $\phi_{I}^{1}$ ) with the isospin projections $I^{\prime}= \pm 1 / 2$ or $\pm 3 / 2, w_{\beta}\left(P, \lambda_{\Delta}\right)$ is the spin $3 / 2$ Rarita-Schwinger spinor-vector with spin projections $\lambda_{\Delta}= \pm 1 / 2, \pm 3 / 2$, and $\psi_{\Delta}(P, k)$ is a scalar wave function. In parallel with the nucleon definition (2.5), we define the wave function with a minus sign. For notational simplicity, the spin indices of the diquark have been omitted from Eq. (2.11) implying that $\varepsilon_{\lambda P}^{\alpha} \rightarrow \varepsilon_{P}^{\alpha}$ with $\lambda=-1,0,+1$. The $\Delta$ wave function therefore consists of three components corresponding to the three different diquark polarizations. These polarizations are summed in the calculation of the transition form factors, as discussed below. The diquark spin polarization vector is, as in the nucleon case, a function of the $\Delta$ mass and momentum. It will be given explicitly in Eqs. (3.2) with $m_{H}=M$.

Since $w_{\beta}\left(P, \lambda_{\Delta}\right)$ are Rarita-Schwinger spinor-vectors, they satisfy the usual constraint conditions [24, 25]

$$
\begin{aligned}
(M-P) w_{\beta}\left(P, \lambda_{\Delta}\right) & =0 \\
P^{\beta} w_{\beta}\left(P, \lambda_{\Delta}\right) & =0 \\
\gamma^{\beta} w_{\beta}\left(P, \lambda_{\Delta}\right) & =0 .
\end{aligned}
$$

Therefore, the wave function (2.11) satisfies the Dirac equation

$$
(M-P) \Psi_{\Delta \lambda_{\Delta}}(P, k)=0,
$$

showing that $\Psi_{\Delta \lambda_{\Delta}}(P, k)$ has no lower (negative energy) components in its rest system.

The isospin wave function $\tilde{\phi}_{I}$, can be written as

$$
\tilde{\phi}_{I^{\prime}}=\left(T \cdot \xi^{1 *}\right) \tilde{\chi}^{I^{\prime}}
$$

where $\xi^{1}$ is the isospin vector of the diquark (identical to the one used for the nucleon), $\tilde{\chi}^{I^{\prime}}$ is a $4 \times 1$ isospin state, and $T^{i}$ denotes the $2 \times 4$ matrix corresponding to the $3 / 2 \rightarrow 1 / 2$ isospin transition operator. The specific form of these operators is given in Appendix B.

As in the nucleon case, $\psi_{\Delta}$ can be expressed as a function of

$$
\chi_{\Delta}=\frac{\left(M-m_{s}\right)^{2}-(P-k)^{2}}{M m_{s}} .
$$

In particular, we use phenomenological ansatz

$$
\psi_{\Delta}(P, k)=\frac{N_{1}}{m_{s}\left(\alpha_{1}+\chi_{\Delta}\right)\left(\alpha_{2}+\chi_{\Delta}\right)^{2}},
$$

where $\alpha_{i}(i=1,2)$ are range parameters in units of $M m_{s}$ and $N_{1}$ a normalization constant. We note that 
the power of $\chi_{\Delta}$ in the denominator differs from the corresponding one in the phenomenological ansatz for the nucleon case. In Appendix G we show that this choice for the $\Delta$ wave function, together with the nucleon wave function (2.9), give the expected $1 / Q^{4}$ pQCD limit for the dominant form factors of the electromagnetic $N \rightarrow \Delta$ transition.

\section{Covariant spin projection operators}

Both the nucleon and $\Delta$ wave functions have a generic structure

$$
\Psi_{H \lambda_{H}}=\phi_{H}(P, k) \varepsilon_{P}^{\alpha *} V_{H \alpha}\left(P, \lambda_{H}\right)
$$

where $H=N, \Delta$, and

$$
\begin{aligned}
V_{H \alpha}\left(P, \lambda_{H}\right) & = \begin{cases}U_{\alpha}\left(P, \lambda_{N}\right) & \text { nucleon } \\
w_{\alpha}\left(P, \lambda_{\Delta}\right) & \Delta\end{cases} \\
\phi_{H}(P, k) & = \begin{cases}-\frac{1}{\sqrt{2}} \psi_{N}(P, k) \phi_{I}^{1} & \text { nucleon } \\
-\psi_{\Delta}(P, k) \tilde{\phi}_{I^{\prime}} & \Delta .\end{cases}
\end{aligned}
$$

Furthermore, in both cases

$$
P^{\alpha} V_{\alpha}\left(P, \lambda_{H}\right)=0 .
$$

These similarities allow us to make some interesting general statements about the nucleon and $\Delta$ wave functions.

The condition (2.19) means that, in the rest frame of the hadron, the vector $V_{\alpha}$ has spatial components only. In this subspace the identity operator is

$$
\mathbf{1}_{\beta}^{\alpha} \equiv \widetilde{g}_{\beta}^{\alpha}=g_{\beta}^{\alpha}-\frac{P^{\alpha} P_{\beta}}{m_{H}^{2}}
$$

where we use the notation $m_{N}=m$ and $m_{\Delta}=M$. This subspace is spanned by two projection operators:

$$
\mathcal{P}_{1 / 2}^{\alpha \beta}+\mathcal{P}_{3 / 2}^{\alpha \beta}=\widetilde{g}^{\alpha \beta}
$$

where, using the notation

$$
\widetilde{\gamma}^{\alpha} \equiv \gamma^{\alpha}-\frac{P P^{\alpha}}{m_{H}^{2}}
$$

the two operators are

$$
\begin{aligned}
& \mathcal{P}_{1 / 2}^{\alpha \beta}=\mathcal{P}_{1 / 2}^{\alpha \beta}(P)=\frac{1}{3} \widetilde{\gamma}^{\alpha} \widetilde{\gamma}^{\beta} \\
& \mathcal{P}_{3 / 2}^{\alpha \beta}=\mathcal{P}_{3 / 2}^{\alpha \beta}(P)=\widetilde{g}^{\alpha \beta}-\frac{1}{3} \widetilde{\gamma}^{\alpha} \widetilde{\gamma}^{\beta} .
\end{aligned}
$$

In Appendix $\mathrm{C}$ we show that these operators are relativistic generalizations of the spin $1 / 2$ and spin $3 / 2$ projection operators (for a particle of mass $m_{H}$ ). They operate only in the $3 \times 3$ subspace of space-like vectors $\widetilde{v}^{\alpha}=v^{\alpha}-P^{\alpha}(P \cdot v) / m_{H}^{2}$. These operators are well known in the literature, in others contexts (the operator $\mathcal{P}_{1 / 2}$ is sometimes denoted $\mathcal{P}_{11}$ ) [26, 27].
As expected, the nucleon and $\Delta$ wave functions are eigenvectors of the spin $1 / 2$ and spin $3 / 2$ operators:

$$
\begin{array}{ll}
\mathcal{P}_{1 / 2}^{\alpha \beta} U_{\beta}=U^{\alpha} & \mathcal{P}_{3 / 2}^{\alpha \beta} U_{\beta}=0 \\
\mathcal{P}_{1 / 2}^{\alpha \beta} w_{\beta}=0 & \mathcal{P}_{3 / 2}^{\alpha \beta} w_{\beta}=w^{\alpha} .
\end{array}
$$

and the orthogonality of these projection operators

$$
\mathcal{P}_{1 / 2}^{\mu \alpha} \mathbf{1}_{\alpha \beta} \mathcal{P}_{3 / 2}^{\beta \nu}=0
$$

implies that the two wave functions are orthogonal, as expected. We will see later that a generalization of this condition is useful in proving current conservation in the electromagnetic $N \rightarrow \Delta$ transition process.

In work already underway [28] these operators will also play an important role in the extension of the formalism to $D$-wave states, for both the nucleon and the $\Delta$.

\section{FIXED-AXIS DIQUARK POLARIZATIONS}

\section{A. Definition of the state vectors}

The spin-1 fixed-axis polarization vectors were introduced in Ref. I, and some of their properties discussed and derived in [14]. (These are really axial vectors, but for simplicity we will drop the word "axial" in the subsequent discussion.) These vectors are denoted $\varepsilon_{\lambda P}^{\mu}$, where $\lambda=0, \pm 1$ is the spin projection in the direction of the baryon three-momentum, $\mathbf{P}$, with $P=\left\{\mathcal{E}_{p}, \mathbf{P}\right\}$ the baryon four-momentum and $\mathcal{E}_{p}=\sqrt{m_{H}^{2}+\mathbf{P}^{2}}$ the baryon energy. In the baryon rest frame the fixed axis may be chosen to be in any direction. Choosing the $\hat{z}$ direction the polarization vectors are

$$
\begin{aligned}
\varepsilon_{0 P_{0}}^{\mu} & =(0,0,0,1) \\
\varepsilon_{ \pm P_{0}}^{\mu} & =\frac{1}{\sqrt{2}}(0, \mp 1,-i, 0) .
\end{aligned}
$$

where the baryon four-momentum in its rest frame is denoted $P_{0}=\left\{m_{H}, 0,0,0\right\}$. These polarization vectors, when used in Eqs. (2.5) and (2.11), give the correct nonrelativistic limit for the nucleon and $\Delta$ wave function.

To write the baryon wave function in a frame where the baryon is moving, a boost in the direction of motion ( $z$-direction by convention) of the baryon is needed. For this choice, $P=\left(\mathcal{E}_{\mathrm{P}}, 0,0, \mathrm{P}\right)$, and the polarization vectors become

$$
\begin{aligned}
\varepsilon_{0 P}^{\mu} & =\frac{1}{m_{H}}\left(\mathrm{P}, 0,0, \mathcal{E}_{\mathrm{P}}\right) \\
\varepsilon_{ \pm P}^{\mu} & =\frac{1}{\sqrt{2}}(0, \mp,-i, 0)
\end{aligned}
$$

and satisfy

$$
\varepsilon_{\lambda P}^{*} \cdot \varepsilon_{\lambda^{\prime} P}=-\delta_{\lambda \lambda^{\prime}}, \quad \varepsilon_{\lambda P} \cdot P=0 .
$$

In the following we will use the variable $P_{-}$for the momentum in the initial state and $P_{+}$for the momentum 
in the final state. Also, the initial state will be a nucleon and the final state will refer either to nucleon or to a $\Delta$.

In the Breit frame, for a transition from an initial state of mass $m$ to a final state of mass $M$, with a momentum transfer $q=P_{+}-P_{-}$, we write

$$
\begin{aligned}
& P_{-}=\left(E_{-}, 0,0,-\frac{1}{2} q_{L}\right) \\
& P_{+}=\left(E_{+}, 0,0, \frac{1}{2} q_{L}\right),
\end{aligned}
$$

where $E_{+}=\sqrt{M^{2}+\frac{1}{4} q_{L}^{2}}$ and $E_{-}=\sqrt{m^{2}+\frac{1}{4} q_{L}^{2}}$ with

$$
q_{L}^{2}=Q^{2}+\frac{\left(M^{2}-m^{2}\right)^{2}}{2\left(M^{2}+m^{2}\right)+Q^{2}} .
$$

Eqs. (3.4) hold for both equal masses $M=m\left(q_{L}=\right.$ $\sqrt{Q^{2}}$ ) and unequal masses.

In the Breit frame, according to Eqs. (3.2), we have for the initial state

$$
\begin{aligned}
& \varepsilon_{0 P_{-}}^{\mu}=\frac{1}{m}\left(-\frac{1}{2} q_{L}, 0,0, E_{-}\right) \\
& \varepsilon_{ \pm P_{-}}^{\mu}=\frac{1}{\sqrt{2}}(0, \mp 1,-i, 0),
\end{aligned}
$$

and for the final state

$$
\begin{gathered}
\varepsilon_{0 P_{+}}^{\mu}=\frac{1}{M}\left(\frac{1}{2} q_{L}, 0,0, E_{+}\right) \\
\varepsilon_{ \pm P_{+}}^{\mu}=\frac{1}{\sqrt{2}}(0, \mp 1,-i, 0) .
\end{gathered}
$$

Note that the polarization vectors $\varepsilon_{P_{ \pm}}^{\mu}$ from Eqs. (3.6)(3.7) refer to the Breit frame only.

Starting from the Breit frame we can then change to an arbitrary frame by means of a suitable Lorentz transformation $\Lambda$. The details of this transformation are discussed in Ref. [14]. The Breit frame momentum $P^{\mu}$ (with $\left.P=P_{ \pm}\right)$is then transformed into $P^{\prime \mu}$ according to

$$
P^{\prime \mu}=\Lambda_{\nu}^{\mu} P^{\nu} .
$$

Due to the four-vector character of $\varepsilon_{P}^{\mu}$, the polarization vectors in the new frame $\varepsilon_{P^{\prime}}^{\mu}$, parametrized by $\Lambda$, become

$$
\varepsilon_{\lambda P^{\prime}}^{\mu}=\Lambda_{\nu}^{\mu} \varepsilon_{\lambda P}^{\nu}
$$

for each polarization $\lambda$. In this notation only the momentum index distinguishes the arbitrary frame (momentum $P^{\prime}$ ) from the Breit frame (momentum $P$ ) polarization vector.

The same transformation $\Lambda$ acts on both final and initial momenta $P_{ \pm}$. The transformation (3.9) combined with the transformation law of the Dirac spinors and the Rarita-Schwinger states (see Appendix B) implies that the baryon wave functions transform as Dirac spinors. The demonstration of the same property for the $\Delta$ wave function follows the lines of the presented in the Ref. 14].

Fixed-axis polarization vectors $\varepsilon_{P}$ are different from the helicity vectors used in Ref. [29], which we denote here by $\eta$. The latter depend on the diquark momentum $k$, and therefore on its direction, satisfying $\eta \cdot k=0$. The helicity vectors $\eta$ can be related to our fixed-axis polarization states by a rotation [14]. In fact, with an appropriate redefinition of the vertex function $\Gamma$, a wave function using fixed-axis states can be made exactly equivalent to another wave function using helicity states. In the case of an initially totally spherical symmetric wave function, the transformation from diquark fixed-axis polarization states to direction-dependent or helicity states gives a vertex function accompanying the helicity vectors just the right angular dependence on the diquark momentum to cancel the dependence introduced by the helicity states $\eta$ [14]. Conversely, a spherically symmetric vertex function $\Gamma$, like the one used here, if taken together with the direction-dependent diquark helicity states $\eta$, would result in a wave function without spherical symmetry. Since here we want to investigate the consequences of spherical symmetric wave functions only, it becomes natural to write these wave functions in terms of fixed-axis diquark polarizations.

\section{B. Importance of the collinearity condition}

We emphasize that matrix elements of states that include fixed-axis polarization vectors must first be constructed in a frame in which the incoming and outgoing states have collinear three-momenta, and only after this has been done can the matrix elements be transformed to an arbitrary frame. If matrix elements are constructed in this order, they will be both unique and covariant, but if they are not constructed in this order, they will be neither covariant nor unique. A simple example of the problems encountered if one does not start with a collinear frame is developed in Appendix D. Our failure to emphasize this point in our original presentation of these ideas lead to a criticism of Kvinikhidze and Miller [30], which we addressed completely in Ref. [14].

Why is a collinear frame required? We will see in the next section that the matrix elements we calculate assume that the diquark is a spectator which does not participate in the interaction. Hence, that the polarization of the diquark emitted by the initial baryon must be the same as the polarization of the diquark absorbed by the final baryon. There are not two distinct diquarks, but one and only one, diquark. Therefore, using fixed-axis polarization states, we must be certain that the polarization states of the diquark emitted from the initial vertex, and of the diquark absorbed into the final vertex, are defined with respect to the same axis, and only in the collinear frame are we certain that the definitions of the polarization of the incoming and outgoing diquark (the same diquark) are consistent with a single direction. Therefore, if we happen to be presented with an interaction in which the initial baryon three-momentum $\mathbf{P}_{-}$is not parallel to the final baryon momentum, $\mathbf{P}_{+}$, we must first transform the matrix element of the collinear frame, 
construct the matrix element, and then transform back to the original frame.

Fortunately, given a any initial and final momentum configuration, there is always a Lorentz transformation that will boost and rotate both states to a collinear frame with the three-momenta $\mathbf{P}_{ \pm}$in the $z$ direction, so the need to define the states in collinear frame imposes no limitation. Our construction is similar to the definition of two-particle helicity states in the two-body center-ofmass by Jacob and Wick [31].

\section{MATRIX ELEMENTS OF THE CURRENT}

\section{A. Definition of the current}

Consider the electromagnetic transition from an initial state $\Psi_{i}(\operatorname{mass} m)$ and a final state $\Psi_{f}(\operatorname{mass} M)$. The relativistic impulse approximation (RIA) to the transition current in the spectator formalism, shown in Fig. 2. is

$$
J_{f i}^{\mu}\left(P_{+}, P_{-}\right)=3 \sum_{\lambda} \int_{k} \bar{\Psi}_{f}\left(P_{+}, k\right) j_{I}^{\mu} \Psi_{i}\left(P_{-}, k\right) .
$$

This is covariant, but we will study it in the collinear Breit frame where the fixed-axis polarizations are consistently defined, as discussed above, and the incoming and outgoing momentum, $P_{\mp}$, are already defined in Eq. (3.4).

In the RIA, the photon couples to each quark through the diagram shown in Fig. 2. The factor of 3 in (4.1) comes from isospin invariance, which allows us to express the sum of the three diagrams in terms of a single integral multiplied by 3 . All intermediate states are taken into account by summing over the diquark spin- 1 polarizations and integrating over all positive on-mass-shell diquark (spectator) states with energy $E_{s}$, using

$$
\int_{k}=\int \frac{d^{3} k}{(2 \pi)^{3} 2 E_{s}} .
$$

There is, in principal, an integration over all spectator masses, $m_{s}$, but this integral is replaced by the value of the integrand at some (unknown) mean value $m_{s}$, which becomes a parameter of the model.

The quark current $j_{I}^{\mu}$ (which is isospin dependent) is decomposed into its Dirac and Pauli terms,

$$
j_{I}^{\mu}=j_{1} \gamma^{\mu}+j_{2} \frac{i \sigma^{\mu \nu} q_{\nu}}{2 m},
$$

where $j_{i}(i=1,2)$ are the quark form factors, defined in Ref. I.

These form factors $j_{1}$ and $j_{2}$ include the quark structure and can be decomposed into isoscalar and isovector components:

$$
\begin{aligned}
& j_{1}=\frac{1}{6} f_{1+}\left(Q^{2}\right)+\frac{1}{2} f_{1-}\left(Q^{2}\right) \tau_{3} \\
& j_{2}=\frac{1}{6} f_{2+}\left(Q^{2}\right)+\frac{1}{2} f_{2-}\left(Q^{2}\right) \tau_{3},
\end{aligned}
$$

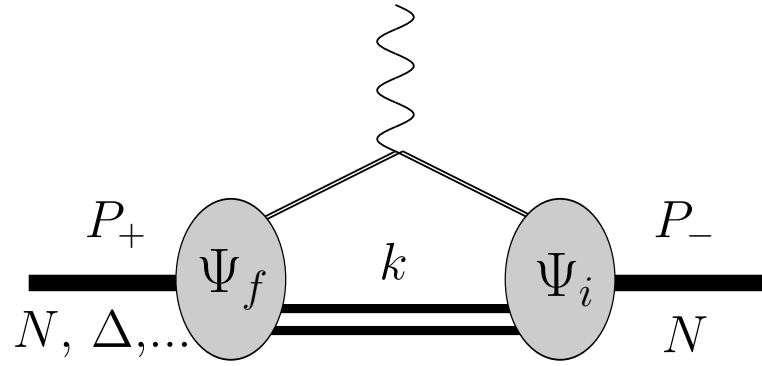

FIG. 2: Relativistic impulse approximation.

where $\tau_{3}$ is the quark isospin operator. In this work we adopted the quark form factors from Ref. [5]

$$
\begin{aligned}
& f_{1 \pm}\left(Q^{2}\right)=\lambda+\frac{(1-\lambda)}{1+Q^{2} / m_{v}^{2}}+\frac{c_{ \pm} Q^{2} / M_{h}^{2}}{\left(1+Q^{2} / M_{h}^{2}\right)^{2}} \\
& f_{2 \pm}\left(Q^{2}\right)=k_{ \pm}\left\{\frac{d_{ \pm}}{1+Q^{2} / m_{v}^{2}}+\frac{\left(1-d_{ \pm}\right)}{1+Q^{2} / M_{h}^{2}}\right\}
\end{aligned}
$$

In these expressions $m_{v}$ and $M_{h}$ are vector meson masses that represent the dominant contributions from the vector dominance model (VDM). The lower mass, $m_{v}=m_{\rho}$ (or $m_{\omega}$ ), describes of the two pion resonance (three pion resonance) effect and $M_{h}$, fixed as $2 m$ (twice the nucleon mass), takes account of all the large mass resonances.

The parameter $\lambda$ is fixed by the deep inelastic scattering (DIS) distribution amplitudes and can interpreted physically as a scaling of the quark charges in DIS limit. All the other parameters are presented in Table 【 They correspond to a previous application of the covariant Spectator theory to the description of the nucleon form factor data with only an $S$-state in the nucleon wave function [5]. We know from the beginning that the restriction to orbital $S$-waves is a considerable simplification, baryon ground state to its first resonance, but it is interesting to see exactly what are the consequences of such a simple assumption.

Note that all of these parameters are fixed by the nucleon data: elastic form factors and DIS. The quark electric form factors $f_{1 \pm}$ are normalized to 1 at $Q^{2}=0$, in order to reproduce the quark and nucleon charge. The quark magnetic form factors are normalized by proton and neutron magnetic moments $f_{2+}(0)=\kappa_{+}=1.639$ and $f_{2-}(0)=\kappa_{-}=1.823$; see Ref. [5] for a detailed discussion.

Due to the relation $T_{i}^{\dagger} \tau_{i}=0$ only the isovector components of the current contributes to the $\gamma N \rightarrow \Delta$ transition. Then, in the following discussion we need the isovector current only, which can be written

$$
\left.j_{I}^{\mu}\right|_{\mathrm{v}}=j_{v}^{\mu} \frac{\tau_{3}}{2}=\left\{f_{1-} \gamma^{\mu}+f_{2-} \frac{i \sigma^{\mu \nu} q_{\nu}}{2 m}\right\} \frac{\tau_{3}}{2},
$$

with the isovector quark form factors $f_{1-}$ and $f_{2-}$ defined as above. 


\begin{tabular}{|c|c|c|c|c|c|}
\hline lod & & $c$ & $d_{+}, d$ &,$m_{s} / r$ & $N_{0}^{2}, \chi^{2}$ \\
\hline \multirow[t]{2}{*}{ I } & 0.057 & 2.06 & -0.444 & 1.22 & 10.87 \\
\hline & 0.654 & 2.06 & -0.444 & 0.88 & 9.26 \\
\hline \multirow[t]{2}{*}{ II } & 0.049 & 4.16 & -0.686 & 0.547 & 11.27 \\
\hline & 0.717 & 1.16 & -0.686 & 0.87 & 1.36 \\
\hline
\end{tabular}

TABLE I: Parameters of the nucleon wave function $\left(\beta_{1}, \beta_{2}\right.$ and $N_{0}^{2}$ ) and quark form factors. In each case we kept $\kappa_{+}=1.639$ and $\kappa_{-}=1.823$ in order to reproduce the nucleon magnetic moments exactly. The difference in the $\chi^{2}$ is mainly due to the description of the neutron electric form factor. Model I preserves the isospin symmetry for the quark electric form factor $f_{1+}=f_{1-}$, but cannot describe neutron electric form factor data. See details in Ref. [5].

\section{B. Diquark polarization sum}

The next step in the general reduction of the transition current is to carry out the sum over the diquark polarizations. Using the generic notation of Eq. (2.17), the current is written

$$
\begin{aligned}
J_{f i}^{\mu}\left(P_{+}, P_{-}\right)= & \frac{3}{2} \int_{k}\left[\phi_{f}\left(P_{+}, k\right) \tau_{3} \phi_{i}\left(P_{-}, k\right)\right] D^{\beta \alpha} \\
& \times \bar{V}_{f \beta}\left(P_{+}, \lambda_{+}\right) j_{v}^{\mu} V_{i \alpha}\left(P_{-}, \lambda_{-}\right),
\end{aligned}
$$

where we assume that only the isovector quark current contributes to form factor (true for the $\gamma N \rightarrow \Delta$ transition) and have written the diquark spin sum as the operator

$$
D^{\mu \nu} \equiv \sum_{\lambda} \varepsilon_{\lambda P_{+}}^{\mu} \varepsilon_{\lambda P_{-}}^{\nu *}
$$

that is evaluated in Ref. [14] and Appendix E The final result shows that $D^{\mu \nu}$ depends only on the momenta and masses of the two states, and can be written

$$
\begin{aligned}
D^{\mu \nu} & =-\left(g^{\mu \nu}-\frac{P_{-}^{\mu} P_{+}^{\nu}}{P_{+} \cdot P_{-}}\right) \\
& +a\left(P_{-}^{\mu}-\frac{P_{+} \cdot P_{-}}{M^{2}} P_{+}^{\mu}\right)\left(P_{+}^{\nu}-\frac{P_{+} \cdot P_{-}}{m^{2}} P_{-}^{\nu}\right),
\end{aligned}
$$

where the factor $a$ is

$$
a=-\frac{M m}{P_{+} \cdot P_{-}\left[M m+P_{+} \cdot P_{-}\right]} .
$$

Note that $D^{\mu \nu}$ satisfies the conditions

$$
P_{+\mu} D^{\mu \nu}=D^{\mu \nu} P_{-\nu}=0 .
$$

\section{Current conservation}

Current conservation requires that $q_{\mu} J_{f i}^{\mu}=0$. To see if this condition is satisfied, we consider separately the Dirac current (from the quark charges) and the Pauli current (from the quark anomalous magnetic moments). The Pauli current is always conserved, independent of the asymptotic states considered. To reduce the Dirac current we use the facts that the initial and final states both satisfy the Dirac equation, and that the charge form factors of the quark depend on $q^{2}$ and can be factored out of the integral

$$
\begin{aligned}
q_{\mu} J_{f i}^{\mu} & =\frac{3}{2} f_{1-} \sum_{\lambda} \int_{k} \bar{\Psi}_{f} \tau_{3} \not \Psi_{i} \\
& =\frac{3}{2}(M-m) f_{1-} \int_{k} \bar{\Psi}_{f} \tau_{3} \Psi_{i}
\end{aligned}
$$

If the masses are equal, the condition is automatically satisfied, but for unequal, masses the states must be orthogonal

$$
\sum_{\lambda} \int_{k} \bar{\Psi}_{f} \tau_{3} \Psi_{i}=0
$$

We can also write Eq. (4.12) using the notation of Eq. (4.7)

$$
\begin{aligned}
q_{\mu} J_{f i}^{\mu}=\frac{3}{2} & (M-m) f_{1-} \int_{k}\left[\phi_{f}\left(P_{+}, k\right) \tau_{3} \phi_{i}\left(P_{-}, k\right)\right] \\
& \times \bar{V}_{f \beta}\left(P_{+}, \lambda_{+}\right) D^{\beta \alpha} V_{i \alpha}\left(P_{-}, \lambda_{-}\right) .
\end{aligned}
$$

For the $\gamma N \rightarrow \Delta$ transition, we can use the projection operators to prove orthogonality. Using the fact that the $N$ and $\Delta$ states are eigenvectors of the spin-1/2 and spin$3 / 2$ projection operators, we can write

$$
\begin{aligned}
& \bar{V}_{\Delta \beta}\left(P_{+}, \lambda_{+}\right) D^{\beta \alpha} V_{N \alpha}\left(P_{-}, \lambda_{-}\right) \\
& =\bar{V}_{\Delta \mu}\left(P_{+}, \lambda_{+}\right)\left[\mathcal{P}_{3 / 2}^{\mu \beta}\left(P_{+}\right) D_{\beta \alpha} \mathcal{P}_{1 / 2}^{\alpha \nu}\left(P_{-}\right)\right] V_{N \nu}\left(P_{-}, \lambda_{-}\right),
\end{aligned}
$$

where $\mathcal{P}_{3 / 2}\left(P_{+}\right)$and $\mathcal{P}_{1 / 2}\left(P_{-}\right)$are the projection operators of Eq. (2.23) with $P \rightarrow P_{+}$and $P \rightarrow P_{-}$, respectively. We show in Appendix $\mathbb{C}$ that the operator in square brackets is zero:

$$
\mathcal{P}_{3 / 2}^{\mu \beta}\left(P_{+}\right) D_{\beta \alpha} \mathcal{P}_{1 / 2}^{\alpha \nu}\left(P_{-}\right)=0
$$

This is the generalization of the orthogonality relation (2.25) and proves the orthogonality of the wave functions for all momentum transfers, $q$. Due to this orthogonality between the initial and the final states, the additional $-\not q q^{\mu} / q^{2}$ term used in the definition of the current in Ref. I vanishes in this application. This is why we did not include that extra term in Eq. (4.3).

\section{V. $\gamma N \rightarrow \Delta$ TRANSITION}

We will now apply the formalism of the previous sections to the study of the electromagnetic $N \Delta$ transition which has a simple interpretation in terms of valence quark structure: the $\Delta$ is a result of a spin flip of a single quark in the nucleon. It is then understandable that 
the magnetic dipole multipole M1 dominates the transition for low $Q^{2}$, while the electric E2 and the Coulomb C2 quadrupoles give contributions of only a few percent. For large $Q^{2}$ however, according to perturbative QCD estimations, we expect equal contributions from M1 and E2 [32]. At the present the $Q^{2}$ scale of the pQCD regime is not known, which motivates calculations within models.

\section{A. Simplification of the transition current}

The transition current (4.7) can now be simplified. Using the notation of Eqs. (2.18) and (4.9) and changing the integration variable from $k$ to $k / m_{s}$ we obtain an integral independent of the diquark mass $m_{s}$ (due to the wave functions normalization which goes with $\left.1 / m_{s}\right)$ and the diquark energy factor $E_{s} / m_{s}=\sqrt{1+k^{2} / m_{s}^{2}}$ (which altogether cancel the $m_{s}^{3}$ dependence of the element $d^{3} k$ ). Factoring out the isospin factors gives

$$
\begin{aligned}
J_{\Delta N}^{\mu}\left(P_{+}, P_{-}\right)= & -\frac{3}{2 \sqrt{2}}\left(\tilde{\phi}_{I^{\prime}}\right)^{\dagger} \tau_{3} \phi_{I}^{1} \int_{k}\left[\psi_{\Delta}\left(P_{+}, k\right) \psi_{N}\left(P_{-}, k\right)\right] \\
& \times \bar{w}_{\beta}\left(P_{+}, \lambda_{+}\right) j_{v}^{\mu} U_{\alpha}\left(P_{-}, \lambda_{-}\right) D^{\beta \alpha}
\end{aligned}
$$

where $j_{v}^{\mu}$ is the defined in terms of the isovector part of the quark current (4.6):

$$
j_{v}^{\mu}=f_{1-} \gamma^{\mu}+f_{2-} \frac{i \sigma^{\mu \nu} q_{\nu}}{2 m},
$$

which is the only part of the quark current to contribute to the transition amplitude. The isospin matrix element is evaluated using the properties of the isospin matrix transition $T^{i}$ (between spin $1 / 2$ states and $3 / 2$ states). Summing over the isospin projections $m_{I}$ of the diquark isospin vector gives

$$
\begin{aligned}
\left(\tilde{\phi}_{I^{\prime}}^{1}\right)^{\dagger} \tau_{3} \phi_{I}^{1} & =-\frac{1}{\sqrt{3}} \tilde{\chi}^{I^{\prime} \dagger} T_{i}^{\dagger} \tau_{3} \tau_{j} \chi^{I} \sum_{m_{I}} \xi_{i}^{1}\left(m_{I}\right) \xi_{j}^{1 *}\left(m_{I}\right) \\
& =-\frac{1}{\sqrt{3}} \tilde{\chi}^{I^{\prime} \dagger}\left(T_{i}^{\dagger} \tau_{3} \tau_{i}\right) \chi^{I}=-\frac{2 \sqrt{2}}{3} \delta_{I I^{\prime}}
\end{aligned}
$$

Next, using the fact that the initial and final states both satisfy the Dirac equation, we may reduce the Pauli form of the current using the Gordon decomposition

$$
\frac{i \sigma^{\mu \nu} q_{\nu}}{2 m}=\gamma^{\mu}\left(\frac{M+m}{2 m}\right)-\frac{P^{\mu}}{m}
$$

where $P^{\mu}$ is the average of the initial and final momentum, defined in Eq. (5.12). We already saw in the discussion of gauge invariance [leading up to the identity [4.16)] that the matrix element of the identity operator is zero, and hence the $P^{\mu}$ term does not contribute. This allows us to collect the quark charge and anomalous magnetic moment contributions into a single term, giving finally

$$
\begin{aligned}
J_{\Delta N}^{\mu}\left(P_{+}, P_{-}\right)= & \delta_{I^{\prime} I} f_{v} \int_{k}\left[\psi_{\Delta}\left(P_{+}, k\right) \psi_{N}\left(P_{-}, k\right)\right] \\
& \times \bar{w}_{\beta}\left(P_{+}, \lambda_{+}\right) \gamma^{\mu} U_{\alpha}\left(P_{-}, \lambda_{-}\right) D^{\beta \alpha},
\end{aligned}
$$

where

$$
f_{v}=f_{1-}+\frac{M+m}{2 m} f_{2-},
$$

is a particular linear combination of the quark from factors that can be factored out of the integral because it depends on $Q^{2}$ only.

Equation (5.5) includes the explicit conservation of the $z$-projection of the isospin. This means that the model predicts that the amplitude is the same for both isospin channels: $\gamma^{*} p \rightarrow \Delta^{+}$and $\gamma^{*} n \rightarrow \Delta^{0}$.

The nucleon wave function $\psi_{N}$ is normalized to one, as required by the charge conservation at $Q^{2}=0$ [5]. Similarly, also the $\Delta$ wave function (2.11) is constrained, in the rest frame where $\bar{P}=(M, 0,0,0)$, by the charge condition (excluding the isospin states from the wave function):

$$
\begin{aligned}
Q_{I} & =3 \sum_{\lambda} \int_{k} \bar{\Psi}_{\Delta}(\bar{P}, k) j_{1} \Psi_{\Delta}(\bar{P}, k) \\
& =\left(\frac{1+\bar{T}_{3}}{2}\right) \int_{k}\left|\psi_{\Delta}(\bar{P}, k)\right|^{2},
\end{aligned}
$$

where $j_{1}=\frac{1}{6}+\frac{1}{2} \tau_{3}$. The isospin operator $\bar{T}_{3}$ is defined as

$$
\bar{T}_{3}=3 \sum_{i} T_{i}^{\dagger} \tau_{3} T_{i}=\left[\begin{array}{rrrr}
3 & 0 & 0 & 0 \\
0 & 1 & 0 & 0 \\
0 & 0 & -1 & 0 \\
0 & 0 & 0 & -3
\end{array}\right] .
$$

Equation (5.7) gives the correct $\Delta$ charge if

$$
\int_{k}\left|\psi_{\Delta}(\bar{P}, k)\right|^{2}=1 .
$$

This condition determines also the normalization constant for the wave function in Eq. (2.16).

\section{B. Form factors: Generalities}

The $N \rightarrow \Delta$ electromagnetic transition current (excluding the electron charge $e$ and ignoring the polarizations of the nucleon and the $\Delta$ ) is given by

$$
J^{\mu}=\bar{w}_{\beta}\left(P_{+}\right) \Gamma^{\beta \mu}(P, q) \gamma_{5} u\left(P_{-}\right),
$$

where the general form of the transition vertex $\Gamma^{\beta \mu}$ is

$$
\Gamma^{\beta \mu}(P, q)=q^{\beta} \gamma^{\mu} G_{1}+q^{\beta} P^{\mu} G_{2}+q^{\beta} q^{\mu} G_{3}-g^{\beta \mu} G_{4} .
$$

The variables $P$ and $q$ are respectively the average of baryon momenta and the photon momentum:

$$
\begin{aligned}
P & =\frac{1}{2}\left(P_{+}+P_{-}\right) \\
q & =P_{+}-P_{-} .
\end{aligned}
$$


The form factors $G_{i}, i=1, \ldots, 4$ depend only on $Q^{2}=$ $-q^{2}$. Due to current conservation, $q_{\mu} \Gamma^{\beta \mu}=0$, only three of the four form factors are independent. In particular, we can write $G_{4}$ in terms of the first three form factors

$$
G_{4}=(M+m) G_{1}+\frac{1}{2}\left(M^{2}-m^{2}\right) G_{2}-Q^{2} G_{3},
$$

and adopt the structure originally proposed by Jones and Scadron 33].

The parametrization (5.11) is not directly comparable to experimental data. More convenient for that purpose are the magnetic dipole (M), electric quadrupole (E) and Coulomb quadrupole (C) form factors defined as

$$
\begin{aligned}
G_{M}^{*}\left(Q^{2}\right)=\kappa\{ & {\left[(3 M+m)(M+m)+Q^{2}\right] \frac{G_{1}}{M} } \\
& \left.+\left(M^{2}-m^{2}\right) G_{2}-2 Q^{2} G_{3}\right\} \\
G_{E}^{*}\left(Q^{2}\right)=\kappa\{ & \left(M^{2}-m^{2}-Q^{2}\right) \frac{G_{1}}{M} \\
& \left.+\left(M^{2}-m^{2}\right) G_{2}-2 Q^{2} G_{3}\right\} \\
G_{C}^{*}\left(Q^{2}\right)=\kappa\{ & 4 M G_{1}+\left(3 M^{2}+m^{2}+Q^{2}\right) G_{2} \\
+ & \left.2\left(M^{2}-m^{2}-Q^{2}\right) G_{3}\right\},
\end{aligned}
$$

where

$$
\kappa=\frac{m}{3(M+m)} .
$$

The three form factors $G_{a}^{*}(a=M, E, C)$ are related to the magnetic, electric, and Coulomb (or scalar) multipole transitions, respectively.

\section{Form Factors: Application of the model}

Substituting for $U_{\beta}$ in (5.5), and suppressing the isospin conservation factor $\delta_{I^{\prime} I}$, gives immediately

$$
J_{\Delta N}^{\mu}\left(P_{+}, P_{-}\right)=\bar{w}_{\beta}\left(P_{+}, \lambda_{+}\right) \mathcal{O}^{\beta \mu} \gamma^{5} u\left(P_{-}, \lambda_{-}\right),
$$

where

$$
\mathcal{O}^{\beta \mu}=\frac{1}{\sqrt{3}} f_{v} \int_{k}\left[\psi_{\Delta}\left(P_{+}, k\right) \psi_{N}\left(P_{-}, k\right)\right] \gamma^{\mu} D^{\beta \alpha} \gamma_{\alpha}
$$

This is easily reduced; the work is given in Appendix F. The final results for the form factors of a transition between $S$-wave nucleon and $\Delta$ states are

$$
\begin{aligned}
G_{M}^{*}\left(Q^{2}\right) & =\frac{8}{3 \sqrt{3}} \frac{m}{(M+m)} f_{v} \mathcal{I} \\
G_{E}^{*}\left(Q^{2}\right) & =G_{C}^{*}\left(Q^{2}\right)=0,
\end{aligned}
$$

where

$$
\mathcal{I}=\int_{k} \psi_{\Delta}\left(P_{+}, k\right) \psi_{N}\left(P_{-}, k\right) .
$$

Note that $\mathcal{I}$ is the only factor which depends on the scalar wave functions and it is Lorentz scalar (frame independent).

\section{RESULTS}

Before we present the numerical results we focus on the analytical structure of the results (5.20)-(5.21). Note that the electric and Coulomb quadrupole form factors vanish in this calculation, which is restricted to $S$-wave orbital states. This result is consistent with quark models based on quark $S$-wave states [13]. According to the literature, the presence of multipoles E2 and C2 is a signature of the nucleon and/or $\Delta$ deformation. Our result of identically vanishing quadrupole form factors is the consequence of considering only $S$-states for both nucleon and $\Delta$ wave functions, and consequently a nucleon and a $\Delta$ with spherical form. The inclusion of higher orbital momentum components would generate non-vanishing electric and Coulomb quadrupoles, as we will confirm in forthcoming work. It is worth noticing that the experimental results for the transition multipoles indicate a contribution of $\mathrm{E} 2$ and $\mathrm{C} 2$ of the order of a few percent at $Q^{2}=0$, consistent with a small angular momentum component in the wave function.

Next, look at the magnetic form factor, $G_{M}^{*}$, at $Q^{2}=0$. Substituting for $f_{v}$ gives

$$
G_{M}^{*}(0)=\frac{2}{3 \sqrt{3}}\left(\frac{2 m}{M+m}+\kappa_{-}\right) \mathcal{I}(0) .
$$

The isovector magnetic moment $\kappa_{-}$was fixed by the nucleon magnetic moment in Ref. [5]

$$
\kappa_{-}=\frac{3}{5}\left(\mu_{p}-\mu_{n}\right)-1=1.823,
$$

For future discussion we write Eq. (6.1) as

$$
G_{M}^{*}(0)=\frac{2}{\sqrt{3}}\left[\frac{\mu_{p}-\mu_{n}}{5}-\frac{1}{3} \frac{M-m}{M+m}\right] \mathcal{I}(0) .
$$

When the experimental nucleon magnetic moment is used in (6.1) one has

$$
G_{M}^{*}(0)=2.07 \mathcal{I}(0)
$$

where

$$
\mathcal{I}(0)=\left.\int_{k} \psi_{\Delta} \psi_{N}\right|_{Q^{2}=0} .
$$

Note, however, that we are working with normalized wave functions

$$
\left.\int_{k}\left|\psi_{N}\right|^{2}\right|_{Q^{2}=0}=1,\left.\quad \int_{k}\left|\psi_{\Delta}\right|^{2}\right|_{Q^{2}=0}=1 .
$$

Because of this conditions, the integral $\mathcal{I}(0)$ is limited, in its absolute value, by the Hölder inequality, the version of the Cauchy-Schwartz inequality for an integral

$$
\left|\int_{k} \psi_{\Delta} \psi_{N}\right| \leq \sqrt{\int\left|\psi_{\Delta}\right|^{2}} \sqrt{\int\left|\psi_{N}\right|^{2}} .
$$


In particular for $Q^{2}=0$ we obtain

$$
|\mathcal{I}(0)| \leq 1
$$

Choosing the positive sign (consistent with our definitions of the scalar wave functions) we conclude that

$$
G_{M}^{*}(0) \leq 2.07 \text {. }
$$

Since the experimental value is considerably larger than this limit,

$$
G_{M}^{*}(0)=3.02 \pm 0.03,
$$

we see that the Spectator quark model, in impulse approximation, can, at best, only describe $69 \%$ of the $\gamma N \rightarrow \Delta$ transition form factor $G_{M}^{*}$ at the photon point.

This underestimation of $G_{M}^{*}(0)$ is an universal property of all constituent quark models. It has been previously reported in the literature [34, 35, 36, 37, 38, 39, 40]. Naive $S$-wave non-relativistic quark models with $S U(6)$ symmetry (and no dynamical effects included) [13], predict

$$
G_{M}^{*}(0)=\frac{2 \sqrt{2}}{3} \sqrt{\frac{m}{M}} \mu_{p}=2.30 .
$$

Including kinematic effects, Ref. [4] obtains

$$
G_{M}^{*}(0)=\frac{2 \sqrt{2}}{3} \frac{\sqrt{2 E_{N}\left(m+E_{N}\right)}}{m+M} \sqrt{\frac{m}{M}} \mu_{p}=2.04,
$$

where $E_{N}$ is the nucleon energy at threshold in the $\Delta$ rest frame. For a review of the constituent quark models predictions see Ref. [13].

Our model for a quark-diquark system differs from other quark models; our quarks are not static, as in Eq. (6.10), and our magnetic form factor is related to both $\mu_{p}$ and $\mu_{n}$, not only to $\mu_{p}$ as in Eq. (6.11). Nevertheless, comparing Eqs. (6.3) and (6.11) we can see that very different descriptions can lead to the similar results if the same constraints are considered (normalization of the wave functions). For completeness we add that calculations based on QCD sum rules also lead to an underestimation of $G_{M}^{*}(0)$ although these models do not apply to the $Q^{2}=0$ region [42]. Similar results are obtained using Generalized Parton Distributions (GDP) [43, 44, 45]. These models extrapolate the Parton Distribution Functions from Deep Inelastic Scattering to intermediate energies. For low $Q^{2} G_{M}^{*}$ is underestimated by $20-30 \%$ [44, 45].

The failure of quark models to describe the $\gamma N \rightarrow \Delta$ transition at threshold shows their limitations, which stem from taking constituent quarks as the only relevant degrees of freedom. Quark wave functions can be normalized to correctly describe nucleon and $\Delta$ static charges, but fail in the description of the dynamical $\gamma N \rightarrow \Delta$ transition which does not involve a charge density, but instead a transition charge density. The magnitude of the difference between the quark model result, which we label the Bare result, and the experimental result, may be due to pion field contributions, and is a manifestation of the strong correlation between the $\Delta$ and the $\pi \mathrm{N}$ system.

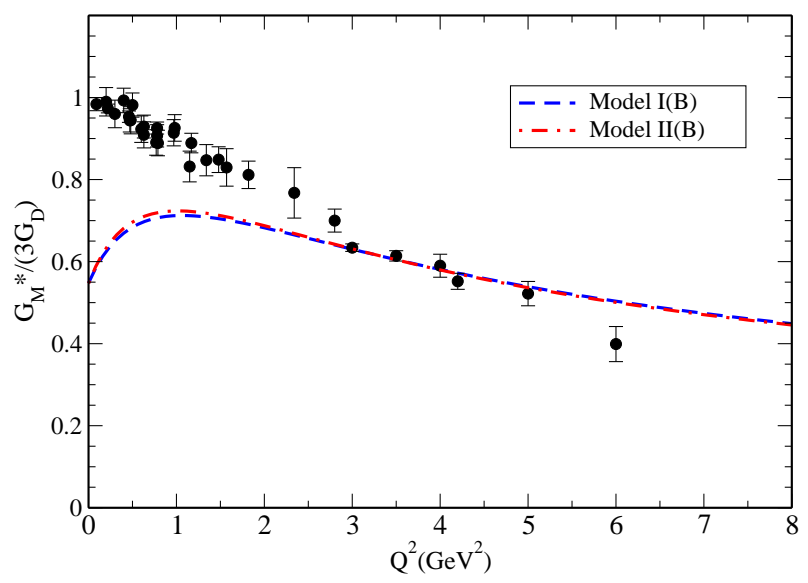

FIG. 3: Result of the fit the $\Delta$ wave function parameters to the data $Q^{2} \geq 2.9 \mathrm{GeV}^{2}$ (where $G_{M}^{\pi}$ is expected to be very small). The nucleon and quark parameters are given by model I and II (see Table I). Delta parameters are presented in Table II] Data from CLAS/Jlab [1, 12], DESY [53] and SLAC [54].

\section{A. Decomposition into Bare and Pion Cloud form factors}

Following the previous discussion, we decompose $G_{M}^{*}$ into two contributions:

$$
G_{M}^{*}\left(Q^{2}\right)=G_{M}^{B}\left(Q^{2}\right)+G_{M}^{\pi}\left(Q^{2}\right)
$$

The term $G_{M}^{B}$ is the Bare form factor: the contribution of the quark core given by the quark model under consideration. The term $G_{M}^{\pi}$ is a contribution due to the pion field: the contribution from any diagram involving a photon and pion loops. Our spectator quark model can predict $G_{M}^{B}$ only.

There are two kinds of descriptions that take into account the effects of the pion field explicitly: dynamical models and low momentum Effective Field Theories or Chiral Perturbation Theories. Here we focus on dynamical models [48, 49, 50] because these models can be used to describe the entire momentum region over which data is available.

A dynamical model uses hadronic degrees of freedom and a coupled channel method to derive transition amplitudes involving initial and final meson-baryon and photon-baryon states. The transition amplitude can be decomposed in two components: (i) the background or non-resonant amplitude which is the solution of an Lippmann-Schwinger-like equation with a nonresonant interaction kernel; and (ii) the resonant amplitude which includes the contributions from dressed intermediate baryon resonance states. The non-resonant interaction kernel that generates the non-resonant background includes direct couplings of the photon or mesons 
with the baryons, and may also include meson rescattering described by intermediate vector mesons. The resonant part is generated by dressing the $s$-channel pole terms generated by vertex functions describing the couplings of photons or mesons to the bare baryon pole. Both the non-resonant direct coupling terms and the resonance vertex functions are parametrized by simple phenomenological expressions with parameters adjusted to fit the pion-nucleon and pion photo-production data. A review can be found in Refs. [13, 46, 47].

The effective contribution of the pion cloud depends on the particular model. The models of Sato and Lee (SL) [48], Dubna-Mainz-Taipei (DMT) [49] predict that the pion cloud will give an important contribution at $Q^{2}=0$ that falls quickly with increasing $Q^{2}$. The Utrecht-Ohio model [50] in opposition predicts small contributions for $Q^{2} \approx 0$ and more significant contributions of $Q^{2} \sim 2$ $\mathrm{GeV}^{2}$ for the pion cloud. According to Ref. [51], pion cloud effects give $33 \%$ of the total contribution at $Q^{2}=0$, and less than $10 \%$ for $Q^{2}>4 \mathrm{GeV}^{2}$.

Pure quark models include no pion cloud effects and can give a complete description only at higher $Q^{2}$ where contributions from the pion cloud are negligible. Supporting this interpretation, our numerical calculations show that we can fit the region $Q^{2}>2.5 \mathrm{GeV}^{2}$, but not the low $Q^{2}$ region. A fit to the higher $Q^{2}$ data $\left(Q^{2} \geq 2.9\right.$ $\left.\mathrm{GeV}^{2}\right)$ is presented in Fig. 3. The $\Delta$ wave function parameters obtained from the two fits shown, referred to as Models I(B) and II(B), are given in Table II. We omitted from this Table the parameters of the nucleon wave function model which also enters the calculation, since that wave function was already fixed by the nucleon form factors and DIS results [5], and those parameters were shown already in Table 1

From the figure we conclude that with no explicit pion cloud we can explain about $55 \%$ of $G_{M}^{*}$ at $Q^{2}=0$. This contribution is lower that the upper limit of Eq. (6.4). For each model, the theoretical quantity $\mathcal{I}(0)$ is a measure of the extent to which a model approaches its theoretical upper limit, and the deviation of $G_{M}^{*}(0) / 3$ from the experimental value 1 is a measure of the quality of the fit to the data at $Q^{2}=0$.

To compare our model with the data over the entire range of $Q^{2}$, we need a parametrization for $G_{M}^{\pi}$. Based on the magnitude of the effects in the DMT model and particularly in the SL model [51], we used a very simple double dipole approximation for the pion cloud

$$
\frac{G_{M}^{\pi}}{3 G_{D}}=\lambda_{\pi}\left(\frac{\Lambda_{\pi}^{2}}{\Lambda_{\pi}^{2}+Q^{2}}\right)^{2}
$$

where $G_{D}=1 /\left(1+Q^{2} / 0.71\right)^{2}\left(\right.$ with $Q^{2}$ in $\left.\mathrm{GeV}^{2}\right)$ and $\lambda_{\pi}$ and $\Lambda_{\pi}^{2}$ are parameters to be adjusted to the data. The parameter $\lambda_{\pi}$ can be interpreted as the fraction of pion cloud effects at $Q^{2}=0$ and $\Lambda_{\pi}^{2}$ measures the falloff of the pion cloud. Note that we parametrize the ratio of $G_{M}^{\pi}$ to $3 G_{D}$ following the tradition of scaling $G_{M}^{*}$ with the dipole factor $G_{D}$. This form assume a falloff of $G_{M}^{\pi} \sim 1 / Q^{8}$, to be compared with $G_{M}^{*} \simeq 3 G_{D} \sim 1 / Q^{4}$.

\begin{tabular}{ccccc} 
Model & $\alpha_{1}, \alpha_{2}$ & $\lambda_{\pi}, \Lambda_{\pi}^{2}$ & $N_{1}, \mathcal{I}(0)$ & $G_{M}^{*}(0) / 3, \chi^{2}$ \\
\hline I(B) & 0.169 & - & 2.88 & 0.548 \\
& 0.489 & - & 0.792 & $\mathbf{1 . 3 2}$ \\
\hline II(B) & 0.181 & - & 3.05 & 0.547 \\
& 0.493 & - & 0.790 & $\mathbf{1 . 2 6}$ \\
\hline I & 0.313 & 0.474 & 2.88 & 1.026 \\
& 0.374 & 1.172 & 0.798 & $\mathbf{2 . 6 4}$ \\
\hline II & 0.290 & 0.464 & 2.95 & 1.012 \\
& 0.393 & 1.224 & 0.794 & $\mathbf{1 . 8 4}$ \\
\hline
\end{tabular}

TABLE II: The dimensionless Delta wave function parameters $\alpha_{1}$ and $\alpha_{2}$ and the normalization constant $N_{1}$ are defined in Eq. (2.16). Model I(B) and II(B) include no pion cloud; Models I and II include a pion cloud with parameters $\lambda_{\pi}$ and $\Lambda_{\pi}^{2}$ (in $\mathrm{GeV}^{2}$ ) defined in Eq. (6.13). The overlap integral between nucleon and Delta wave-function (which cannot be larger than 1) is $\mathcal{I}(0) ; G_{M}^{*}(0) / 3$ measures the quality of the fit for $Q^{2}=0$ (where the experimental result $\simeq 1$ ).

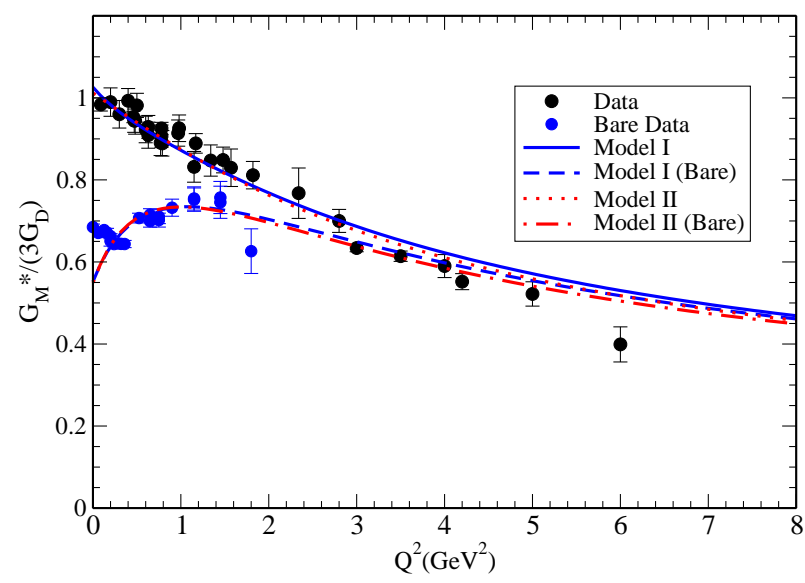

FIG. 4: Fit to the $G_{M}^{*}$ and Bare data using using model I and II. The Bare data is from [41, 52], $G_{M}^{*}$ data from figure 3. The Bare result is now defined considering $\lambda_{\pi}=0$ in the dressed models.

It is important to realize that, due to the complexity of the pion production process, the decomposition of Eq. (6.12) is strongly model dependent. Dynamical models can differ in coupling constants, off-mass-shell extrapolations, and off course the parametrization of bare component itself. Using Eq. (6.13) we can find several combinations $\left(G_{M}^{B}, G_{M}^{\pi}\right)$ with approximately the same sum $G_{M}^{*}$. We must find some way to constrain one of these two components.

To do this, we use a procedure implemented for the first time in Ref. [41]. Using the SL model, Julia-Diaz, Lee, Sato and Smith extract, independently at each $Q^{2}$ point, a value of the bare form factor. This is possible because the bare form factor, $G_{M}^{B}$, is one of the parameters that enters the dynamical SL model, and it is therefore 
possible to determine it, without any theoretical bias, by a best fit to the data.

The data for the bare component of the form factor, determined in this way, are shown in Fig. 4. Note that a separation between the "bare" data and the experimental data can only be made in the region $Q^{2}<2.5 \mathrm{GeV}^{2}$. Above $Q^{2} \sim 3 \mathrm{GeV}^{2}$ the pion cloud contributions are less significant and the dynamical model produces only a correction to the bare component; in this region the full contribution comes mainly from the bare component.

Models I and II, dressed by the pion cloud, are a result of a simultaneous fit of both components of the form factor, Eq. (6.12), to the $G_{M}^{*}$ experimental data [11, 12, 53, 54 and of the bare component, to the bare "data" for $Q^{2}<2 \mathrm{GeV}^{2}$ based on the SL extraction [41, 52] discussed above. The parameters for Models I and II are compared to models I(B) and II(B) in Table (The $\chi^{2}$ given there is for the fit to $G_{M}^{*}$ only.) In Fig. 4 we present the results our predictions for the dressed models and the respective Bare version obtained setting $\lambda_{\pi}=0$. In the same figure we can see that the data below $0.13 \mathrm{GeV}^{2}$ (first three points) cannot be described by our model. This limitation is related to the behavior of the overlap integral of the nucleon and Delta wave functions. Note that the values of $\mathcal{I}(0)$ are almost identical for the bare and dressed models, but the dressed models now have $G_{M}^{*}(0) / 3 \approx 1$ due to the addition of a pion cloud term of about $46 \%$ at $Q^{2}=0$. Furthermore, a reasonable description of the 'bare data' is obtained for both models $\left(\chi^{2}=4.2\right.$ for model I and $\chi^{2}=4.6$ for model II) at least for $Q^{2}>0.13 \mathrm{GeV}^{2}$.

It is worth to mentioning that the parametrization of the dressed models changes the results of the bare contribution relative to the Fig. 3. Although similar, the bare results presented in Fig. 4 are slightly larger than the results of the models $\mathrm{I}(\mathrm{B})$ and $\mathrm{II}(\mathrm{B})$ presented in Fig. 3 . in particular for $Q^{2}<2 \mathrm{GeV}^{2}$. This increment is not obvious in the graphs, but as we can see in table III, the parameters $\alpha_{1}$ and $\alpha_{2}$ for the models I(B) and II(B) are significantly different from the models I and II. This feature is the result of including the low momentum "bare data" $\left(Q^{2}<2 \mathrm{GeV}^{2}\right)$ that appears not be completely consistent with the high $Q^{2}$ data $\left(Q^{2}>3 \mathrm{GeV}^{2}\right)$ at least with the naive parametrization (6.13). To sort out this situation more high $Q^{2}$ data of high quality are needed (the current data set includes only 6 data points with $Q^{2} \geq 3 \mathrm{GeV}^{2}$, compared with 26 data points for $G_{M}^{*}$ and 21 data points for $G_{M}^{B}$ for $Q^{2}<3 \mathrm{GeV}^{2}$ ).

\section{B. Comparing with other models for the Bare form factors}

As mentioned above, the dynamical models need a phenomenological parametrization of the "bare" vertex. There is some freedom in the choice of the "bare" form factor, but the constraints of the quark models are usually taken into account. This parametrization can be

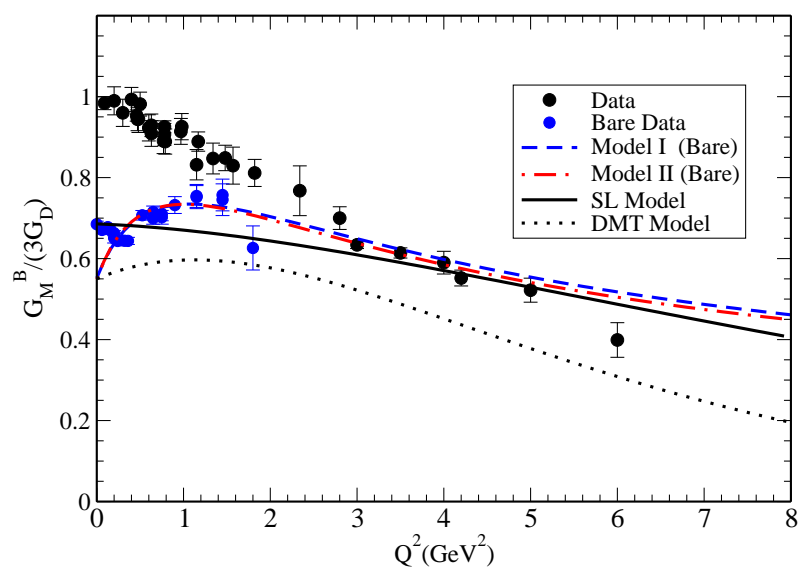

FIG. 5: Comparing Bare form factor parametrization with the 'bare data' from Refs. [41, 52] with models I and II, SL model [48] and DMT model [49].

done for each transition multipole $G_{\alpha}^{*}(\alpha=M, E, C)$. For the SL and DMT models the "bare" form factors can be written

$$
G_{M}^{B}=G_{M}^{B}(0)\left(1+a Q^{2}\right) \exp \left(-b Q^{2}\right) f
$$

where $G_{M}^{B}(0) \leq 2.07$ fixes the contribution of the quark core for $Q^{2}=0, a, b$ are positive parameters, and $f=1$ for the SL model and $f=\sqrt{1+\frac{Q^{2}}{(M+m)^{2}}}$ for the DMT model. For SL $G_{M}^{B}(0)=2$; for DMT $G_{M}^{B}(0)=1.65$. All other parameters can be found in Refs. [48, 49].

The structure of the Utrecht-Ohio model [50] for the bare form factor is incompatible with a pion cloud which is not zero for $Q^{2}=0$, and for this reason a direct and simple estimation of the bare form factor based in Eqs. (6.12) and (6.13) is not possible.

The bare form factors use by of SL and DMT [obtained from the analytical expression (6.14)] and our numerical result for models I and II are compared in Fig. 5. We see that the bare SL form factor overestimates the data for $Q^{2}>4 \mathrm{GeV}^{2}$ and that DMT always underestimates the data (suggesting a significant pion cloud contribution even for $Q^{2}>3 \mathrm{GeV}^{2}$, since for $4 \mathrm{GeV}^{2}$ the effect is still about 10\%). We need to point out that the particular parametrization of both SL and DMT, in particular the SL model, was done before the data $Q^{2}>4 \mathrm{GeV}^{2}$ became available [12]. The new data showed the limitation of the particular parametrization, and was one of the motivations for work presented in Ref. 41], where the Bare form factor are adjusted for each $Q^{2}$ point. Note that our results are very similar to SL for $Q^{2}>3 \mathrm{GeV}^{2}$. 


\section{Comparing Model I with Model II}

In this work we consider two different models for the nucleon wave function as presented in Ref. [5]. We conclude that the model II is the best model for the $\gamma N \rightarrow \Delta$ transition because is the one that better describes the high $Q^{2}$ data (meaning the pion cloud contribution is better described by the dipole form). Model I gives a slightly worse description of the data, but both models are almost indiscernible in the region $Q^{2}<3 \mathrm{GeV}^{2}$. This result is very interesting because the models are fundamentally different in the description of the nucleon form factors. In the model I the isospin symmetry is exactly imposed with $f_{1+}=f_{1-}$ (Eq. (4.5) with $c_{+}=c_{-}$), leading to the failure of the description of the neutron electrical form factor (see Ref. [5] ) and a consequent high $\chi^{2}$ penalization as shown in table I. As the $\gamma N \rightarrow \Delta$ transition is independent of $f_{1+}$ (only isovector form factors contribute) a reasonable description of the form factors can be obtained for both models.

\section{Discussion}

Since our model includes only $S$-waves in the nucleon and $\Delta$ wave functions, the comparison of our results to all models and frameworks used in the description of the electromagnetic $N \rightarrow \Delta$ transition has to be done with care. As mentioned above, we can only predict non-zero contributions to the dominant form factor $G_{M}^{*}$ (55\% of it for $Q^{2}=0$ ), while the experiments reveal two more non-vanishing, albeit small, form factors $\left(G_{E}^{*}\right.$ and $\left.G_{C}^{*}\right)$. The limitation of our model is visible in its failure at high $Q^{2}$, where $G_{E}^{*}$ is comparable with $-G_{M}^{*}$, according to $\mathrm{pQCD}$. That regime is however out of reach of the present state-of-the-art measurements.

On the other hand, our results are hardly comparable with the low momentum Effective Field Theory and Chiral Perturbation Theory [55, 56, 57, 58, 59]. Those models include pion degrees of freedom consistently at least at one pion loop level. But the range of the prediction is limited to $Q^{2}<0.25 \mathrm{GeV}^{2}$, due to the expansion in terms of the terms of the small variables (pion momentum, pion mass, difference of $\Delta$ and nucleon mass) - Besides the range limitation, the bare contribution is adjusted to the data around $Q^{2}=0$ and is not really predicted from quark structure. This leads to a bare contribution significantly different from quark models. Note that Effective Chiral Perturbation Theory relies on an energy scale parameter, usually $\lambda \sim 1 \mathrm{GeV}$, decoupling the short range physics (bare) from the long range physics where the pion cloud is included. In Ref. [57] the pion cloud gives a positive contribution; in Ref. [59] the contribution is negative (bare contribution $G_{M}^{B}(0)=4.04$ ).

As for lattice QCD data, the comparison is not yet conclusive: the (quenched) lattice data of Alexandrou et al [60] overpredict $G_{M}^{*}$ in the chiral limit for $Q^{2}>0.1$ $\mathrm{GeV}^{2}$. In principle, the quenched lattice data should be comparable to the bare form factor results, and hence it might be expected to be larger that the experimental data, but instead it underestimates the data. It is not known yet either this discrepancy is due to the limitations of the quenched data or to the extrapolation to the physical region. The available results from full QCD (unquenched) are not adequate for an extrapolation [61].

The soundest comparison to be made, then, is to other valence quark models. This is why in the previous subsection we compared the magnitude of the Bare form factor obtained by us to the results from dynamical models and constituent quark models based on $S$-wave wave functions. We are left then with comparing our results to the predictions of Light-Cone Sum Rules of Braun et al 42]. This formalism divides the main contributions of the form factors into two components: the soft contribution falling with $1 / Q^{6}$ and the hard contribution due to pQCD with a $1 / Q^{4}$ falloff. The soft contributions, dominant in the intermediate region, are explicitly evaluated, using the nucleon asymptotic amplitudes (valence quark distributions), and an adjustable momentum range parameter (Borel parameter). Their results describe $G_{M}^{*}$ in the $3-6 \mathrm{GeV}^{2}$ region, but fail in the region of low and high $Q^{2}$ (where they have an almost constant slope). Also they underestimate $G_{M}^{*}$ at low $Q^{2}$ like the constituent quark models (at $Q^{2}=1 \mathrm{GeV}^{2}$ the prediction for $G_{M}^{*}$ only takes account of $60 \%$ of the experimental value).

The success of our model in the description of the bare form factors (high $Q^{2}$ region) is related to our choice for the form of $\psi_{\Delta}$ in Eq. (2.16). In particular, the extra power in the $\alpha_{2}+\chi_{\Delta}$ factor, comparatively to the nucleon wave function, plays a key role. It is also interesting to note that our results would be very similar to the results of the Ref. 42] had we used a $\Delta$ scalar function of the nucleon-type (see Eq. (2.9)). This generates $G_{M}^{*}$ with an almost constant slope and $G_{M}^{*} /\left(3 G_{D}\right) \simeq 0.6$. As mentioned, our parametrization for the $\Delta$ scalar form factor $\psi_{\Delta}$ is consistent with $G_{M}^{*} \sim 1 / Q^{4}$ for large $Q^{2}$, i.e., the pQCD prediction. For the $\Delta \Delta$ form factors (a $\Delta$ in the initial and final state) our prediction, based on Eq. (2.16), has a $1 / Q^{6}$ falloff for the dominant form factors at high $Q^{2}$, instead of the $1 / Q^{4}$ advocated by pQCD. We can find a good compromise for both the low $Q^{2}$ description and the expected pQCD behavior, by considering

$$
\psi_{\Delta}=\frac{a}{\left(\alpha_{1}^{\prime}+\chi_{\Delta}\right)\left(\alpha_{2}^{\prime}+\chi_{\Delta}\right)}+\frac{b}{\left(\alpha_{1}+\chi_{\Delta}\right)\left(\alpha_{2}+\chi_{\Delta}\right)^{2}},
$$

where $\alpha_{i}^{\prime}$ are new range parameters, and $a, b$ coefficients which balance the two regimes: $a$ the $\operatorname{deep} Q^{2}$ asymptotic region and $b$ the low-intermediate $Q^{2}$ region. This compromise requires $b>>a$ pushing the pQCD dominance to very far away.

\section{SUMMARY AND CONCLUSIONS}

We have developed a systematic formalism for the description of baryon wave functions, built upon con- 
stituent quark (flavor, spin and isospin) and baryon (spin, isospin) effective properties. The form of the nucleon and $\Delta$ wave functions with $S$-wave orbital angular momentum components only is presented. The formalism is manifestly covariant. The wave functions are covariant and transform like Dirac and Rarita-Schwinger spinors. The matrix elements are covariant, with the same form in all frames.

One of our models (model II) describes the nucleon form factor data [5] and the dominant contribution to the $N \rightarrow \Delta$ electromagnetic transition. The results for $G_{M}^{*}$ show a reasonable agreement with the data, and explain its measured falloff. They are also consistent with the long range behavior predicted by pQCD $\left(G_{M}^{*} \simeq 1 / Q^{4}\right)$ [32]. Our results for $G_{M}^{*}$ are consistent with the results of quark models where only $S$-states are considered.

In agreement with previous works (see Refs. 13, 41, 42, 46]), we conclude that a successful description of $G_{M}^{*}$ near $Q^{2}=0$ requires an addition of a pion cloud term not included in the class of valence quarks to explain the strength at $Q^{2}=0$ of the magnetic form factor $G_{M}^{*}$ of the $N \rightarrow \Delta$ electromagnetic transition. Our predictions for the pion cloud underestimate the predictions based on the Sato and Lee model (46\% versus $33 \%$ ) for $Q^{2}=0$. This gap can in principle decrease once $D$-states are included into the $\Delta$ wave function. The magnitude of the pion cloud for $Q^{2}=0$ is similar to the estimations of the DMT model but we predict a faster falloff. [Note that our results are not directly compared with the DMT model because our 'bare' contribution is fixed by the 'bare data' extraction of the SL model.] Except for the region $Q^{2} \sim 0$, our model is consistent with the 'Bare data' extraction based in Sato and Lee model [41]. For the region $Q^{2}>3 \mathrm{GeV}^{2}$ our model and the original SL model [48] overestimate the data slightly. In this region either the pion cloud parametrization is not adequate or the data is insufficient to constraint adequately the parameters of the pion cloud. More higher $Q^{2}$ data and more accurate data for both $G_{M}^{*}$ and $G_{M}^{B}$ (SL model) is therefore necessary for to establish the effect of the pion cloud.

Next, we plan to generalize the structure of the wave functions to include higher orbital angular momentum states in the quark-diquark system, without loss of the covariance requirement. The inclusion of $\mathrm{D}$ states in the nucleon and $\Delta$ is in progress.

\section{Acknowledgments}

The authors wants to thank to B. Juliá-Díaz for sharing the 'bare data' of Ref. [41]. This work was partially support by Jefferson Science Associates, LLC under U.S. DOE Contract No. DE-AC05-06OR23177. G. R. was supported by the portuguese Fundação para a Ciência e Tecnologia (FCT) under the grant SFRH/BPD/26886/2006.

\section{APPENDIX A: NUCLEON WAVE FUNCTION}

In the non-relativistic limit, the wave function of a spin $1 / 2$ system composed of a quark (spin 1/2) and a quark pair (diquark), as in the nucleon, can be decomposed into two components: the scalar part (spin-0 diquark) and the axial-vector part (spin-1 diquark).

The spin- 0 part is just

$$
\phi_{s}^{0}=\chi_{s},
$$

where $\chi_{s}$ is the usual Pauli spinor $(2 \times 1$ state). The spin of the system is then given by the spin of the quark. The relativistic generalization is $u\left(P_{0}, s\right)$, where $P_{0}=\{m, \mathbf{0}\}$ is the four-momentum of a nucleon at rest.

The spin-1 component of the wave function $\phi_{s}^{1}$ describes the quark-diquark spin $1 / 2$ system (nucleon) in the initial state and a diquark polarization vector in the final state. The quark-diquark spin state, represented by $V_{N s}$, is a direct product of a spin-1 diquark state with a spin $1 / 2$ quark state. In this case the three-component vector $\varepsilon_{\lambda}^{i}$ (with $i=x, y, z$ and $\lambda=0, \pm$ the diquark polarization index) describes the diquark polarization [these vectors are the three-component parts of the polarization vectors defined in Eqs. [3.1)] . Then

$$
\left(V_{N s}\right)^{i}=\sum_{\lambda s^{\prime}}\left\langle 1 \lambda ; \frac{1}{2} s^{\prime} \mid \frac{1}{2} s\right\rangle \varepsilon_{\lambda}^{i} \chi_{s^{\prime}},
$$

where $s= \pm 1 / 2$ is the spin projection of the nucleon, $\left\langle s_{1} m_{1} ; s_{1} m_{2} \mid j m_{j}\right\rangle$ is the Clebsch-Gordan coefficient that couples spins $s_{1}$ and $s_{2}$ to total spin $j$, and $\chi_{s^{\prime}}$ the quark spinor. Explicitly

$$
\begin{aligned}
& \left(V_{N,+\frac{1}{2}}\right)^{i}=\sqrt{\frac{2}{3}} \varepsilon_{+}^{i} \chi_{-\frac{1}{2}}-\sqrt{\frac{1}{3}} \varepsilon_{0}^{i} \chi_{+\frac{1}{2}} \\
& \left(V_{N,-\frac{1}{2}}\right)^{i}=\sqrt{\frac{1}{3}} \varepsilon_{0}^{i} \chi_{-\frac{1}{2}}-\sqrt{\frac{2}{3}} \varepsilon_{-}^{i} \chi_{+\frac{1}{2}} .
\end{aligned}
$$

Equation (A2) can be written

$$
\left(V_{N s}\right)^{i}=-\frac{1}{\sqrt{3}} \sigma_{i} \chi_{s}
$$

where $\chi_{s}$ now represents the nucleon spinor. The natural relativistic generalization is

$$
\left(V_{N s}\right)^{i} \rightarrow U^{\alpha}\left(P_{0}, s\right)=\frac{1}{\sqrt{3}} \gamma_{5}\left(\gamma^{\alpha}-\frac{P_{0}^{\alpha}}{m}\right) u\left(P_{0}, s\right),
$$

where $u\left(P_{0}, s\right)$ is the Dirac spinor of the nucleon and $\alpha=\{0, i\}$ with $U^{0}=0$.

Note that Eq. (A4) describes only the initial state of the vertex represented in Fig. 1 (the 3-quark bound state). To obtain the amplitude of the full process we need to contract $\left(V_{s}\right)^{i}$ with the diquark final state $\varepsilon_{\lambda}^{\alpha *}$ (the quark is off-shell with its final spin state unspecified). As result, we have the amplitude

$$
\phi_{s}^{1}=-\varepsilon_{\lambda P_{0}}^{\alpha *} U_{\alpha}\left(P_{0}, s\right) .
$$

Equations (A4) and (A5) can both be generalized for a moving nucleon by means of a boost in the $z$-direction. 


\section{APPENDIX B: SPIN STRUCTURE FOR THE $\Delta$ $S$-STATE}

In close analogy with the nucleon, the $\Delta$ wave function, in its rest frame rest frame, can be written as a direct product of a spin-1 diquark and a spin-1/2 quark

$$
\left(V_{\Delta s}\right)^{i}=\sum_{\lambda s^{\prime}}\left\langle 1 \lambda ; \frac{1}{2} s^{\prime} \mid \frac{3}{2} s\right\rangle \varepsilon_{\lambda}^{i} \chi_{s^{\prime}} .
$$

where $s= \pm 3 / 2$ or $\pm 1 / 2$ is the spin projection of the $\Delta$. Once again $\varepsilon_{\lambda}^{i}$ is the diquark polarization in the $\Delta$ rest frame, and $\chi_{s^{\prime}}$ a quark Pauli spinor.

We can also express $V_{\Delta s}$ in terms of a basis of spin $3 / 2$ states:

$$
\begin{aligned}
& \omega_{+\frac{3}{2}}=\left(\begin{array}{l}
1 \\
0 \\
0 \\
0
\end{array}\right) \quad \omega_{+\frac{1}{2}}=\left(\begin{array}{l}
0 \\
1 \\
0 \\
0
\end{array}\right) \\
& \omega_{-\frac{1}{2}}=\left(\begin{array}{l}
0 \\
0 \\
1 \\
0
\end{array}\right) \quad \omega_{-\frac{3}{2}}=\left(\begin{array}{l}
0 \\
0 \\
0 \\
1
\end{array}\right) .
\end{aligned}
$$

In this case the connection between the $\omega$ and $V_{\Delta s}$ can be written

$$
\left(V_{\Delta s}\right)^{i}=\mathcal{T}^{i} \omega_{s}
$$

where $\mathcal{T}^{i}$ is an $2 \times 4$ matrix that transforms the spin $3 / 2$ state of the $\Delta$ into the spin $1 / 2$ state (of a quark). The elements of $\mathcal{T}^{i}$ can be evaluated using the coefficients in Eq. (B1). The result is

$$
\begin{aligned}
\mathcal{T}^{x} & =-\frac{1}{\sqrt{6}}\left(\begin{array}{cccc}
\sqrt{3} & 0 & -1 & 0 \\
0 & 1 & 0 & -\sqrt{3}
\end{array}\right) \\
\mathcal{T}^{y} & =-\frac{i}{\sqrt{6}}\left(\begin{array}{cccc}
\sqrt{3} & 0 & 1 & 0 \\
0 & 1 & 0 & \sqrt{3}
\end{array}\right) \\
\mathcal{T}^{z} & =\sqrt{\frac{2}{3}}\left(\begin{array}{cccc}
0 & 1 & 0 & 0 \\
0 & 0 & 1 & 0
\end{array}\right) .
\end{aligned}
$$

As in Eq. (B1), $\left(V_{\Delta s}\right)^{i}$ is a $2 \times 1$ spinor with a spin $1 / 2$ structure.

To convert (B1) to relativistic form, add a negative energy $4 \times 1$ lower component that vanishes in the $\Delta$ rest frame:

$$
\left(V_{\Delta s}\right)^{i} \rightarrow\left[\begin{array}{c}
\mathcal{T}^{i} \omega \\
0
\end{array}\right] \equiv w^{i}\left(P_{0}, s\right)
$$

Here $w^{i}\left(P_{0}, s\right)$ is the Rarita-Schwinger vector-spinor for 3 -momentum $\mathbf{P}=0$. It satisfies the constraint Eqs. (2.12). These constraints insure that $w^{0}$ vanishes in the rest frame, as implied by Eq. (B5).
To generalize the states to an arbitrary frame with $\mathbf{P} \neq 0$, we boost them using a Lorentz transformation $\Lambda$, giving

$$
w^{\beta}(P, s)=S(\Lambda) \Lambda_{\alpha}^{\beta} w^{\alpha}\left(P_{0}, s\right) .
$$

Using the state $w^{\beta}$ in a arbitrary frame, the $\Delta$-quarkdiquark vertex is constructed in the same way as the nucleon vertex. Considering the final state diquark polarization vector, $\varepsilon_{\lambda}^{*}$, following the nucleon state convention of Eq. (A5)

$$
\tilde{\phi}_{s}^{1}=-\varepsilon_{\lambda P_{0}}^{\beta *} w_{\beta}\left(P_{0}, s\right),
$$

gives the spin wave function introduced of Eq. (2.11).

\section{APPENDIX C: RELATIVISTIC SPIN PROJECTION OPERATORS}

In this paper we work with operators $\mathcal{O}^{\alpha \beta}$ that satisfy the constraint equations

$$
P_{\alpha} \mathcal{O}^{\alpha \beta}=0=\mathcal{O}^{\alpha \beta} P_{\beta}
$$

In the particle rest system, such operators "live" in the $3 \times 3$ subspace corresponding to nonrelativistic 3 dimensional space, and it is easy to relate these operators to their nonrelativistic analogues.

As an example, consider the projection operators that operate on the direct product of spin-1 and spin-1/2 spaces. The total angular momentum operator is the sum

$$
J^{i}=W^{i}+S^{i}
$$

where $W^{i}$ are spin-1 operators (with multiplication by the unit operator on the spin-1/2 space implied) and $S^{i}$ are the spin- $1 / 2$ operators (with multiplication by the unit operator on the spin-1 space implied). The projection operators are constructed from the operator

$$
2 \mathbf{W} \cdot \mathbf{S}=\mathbf{J}^{2}-\mathbf{W}^{2}-\mathbf{S}^{2}=\left\{\begin{array}{rl}
1 & J=\frac{3}{2} \\
-2 & J=\frac{1}{2}
\end{array}\right.
$$

Hence the projection operators $\mathcal{P}_{J}$ are

$$
\begin{aligned}
& \mathcal{P}_{1 / 2}=\frac{1}{3}(1-2 \mathbf{W} \cdot \mathbf{S}) \\
& \mathcal{P}_{3 / 2}=\frac{1}{3}(2+2 \mathbf{W} \cdot \mathbf{S}) .
\end{aligned}
$$

Using $\left(W_{j k}\right)^{i}=-i \epsilon_{i j k}$ and $S^{i}=\sigma^{i} / 2$ we get

$$
\begin{aligned}
& \left(\mathcal{P}_{1 / 2}\right)_{j k}=\frac{1}{3}\left(\delta_{j k}+i \epsilon_{i j k} \sigma^{i}\right)=\frac{1}{3} \sigma_{j} \sigma_{k} \\
& \left(\mathcal{P}_{3 / 2}\right)_{j k}=\delta_{j k}-\frac{1}{3} \sigma_{j} \sigma_{k},
\end{aligned}
$$

leading immediately to the relativistic generalizations

$$
\begin{aligned}
& \left(\mathcal{P}_{1 / 2}\right)^{\alpha}{ }_{\beta}=\frac{1}{3}\left(\gamma-\frac{P_{0} P_{0}}{m_{H}^{2}}\right)^{\alpha}\left(\gamma-\frac{P_{0} P_{0}}{m_{H}^{2}}\right)_{\beta} \\
& \left(\mathcal{P}_{3 / 2}\right)^{\alpha}{ }_{\beta}=g^{\alpha}{ }_{\beta}-\frac{P_{0}^{\alpha} P_{0 \beta}}{m_{H}^{2}}-\left(\mathcal{P}_{1 / 2}\right)^{\alpha}{ }_{\beta} .
\end{aligned}
$$


Boosting these from $P_{0}$ to $P$ gives the operators introduces in Eq. (2.23) above.

References [26, 27] define two spin $1 / 2$ projection operators. In addition to $\mathcal{P}_{1 / 2}$ (which they call $\mathcal{P}_{11}$ ) they introduce the operator

$$
\left(\mathcal{P}_{22}\right)_{\beta}^{\alpha}=\frac{P^{\alpha} P_{\beta}}{M^{2}}
$$

is a spin $1 / 2$ projector on the $\left(0, \frac{1}{2}\right)$ space. Since we work in the space of operators satisfying the constraint (C1), this operator is excluded from our basis. In our formalism, it is part of the operator $\tilde{g}$.

We conclude this appendix by proving the useful relation (4.16). Start by using the properties

$$
P_{+}^{\mu} D_{\mu \nu}=0=D_{\mu \nu} P_{-}^{\nu},
$$

which follow directly from the definition of $D_{\mu \nu}$ as a sum over polarizations. Then we prove two intermediate results. First, using the notation $\mathcal{P}_{1 / 2}^{\mu \alpha}\left(P_{+}\right)$to denote the projection operator with momentum $P_{+}$for a state with mass $M$ [and similarly for $\mathcal{P}_{1 / 2}^{\beta \nu}\left(P_{-}\right)$], we see that

$$
\mathcal{P}_{1 / 2}^{\mu \alpha}\left(P_{+}\right) D_{\alpha \beta} \mathcal{P}_{1 / 2}^{\beta \nu}=\frac{1}{9} \widetilde{\gamma}^{\mu}\left(P_{+}\right)\left[\gamma^{\alpha} D_{\alpha \beta} \gamma^{\beta}\right] \widetilde{\gamma}^{\nu}\left(P_{-}\right) .
$$

If $b=P_{+} \cdot P_{-}$, the quantity in square brackets is

$$
\begin{aligned}
{[\cdots] } & =-4+\frac{\not P_{-} \not P_{+}}{b}+a\left[\not P_{-}-\frac{b}{M^{2}} \not P_{+}\right]\left[\not P_{+}-\frac{b}{m^{2}} \not P_{-}\right] \\
& =-4+\frac{\not P_{-} \not P_{+}}{b}+a\left[\not P_{-}+\frac{b}{M}\right]\left[\not P_{+}+\frac{b}{m}\right] \\
& =-4-2 a b+\frac{a b^{2}}{M m}+\not P_{-} \not P_{+}\left[\frac{1}{b}+a\right], \quad(\mathrm{C} 10)
\end{aligned}
$$

where we used the fact that $\not_{-} \rightarrow-m$ when acting to the right, and $\not_{+} \rightarrow-M$ when acting to the left, because both anticommute with the $\widetilde{\gamma}$ standing to the right and left, and then can be eliminated using the Dirac equation satisfied by the incoming and outgoing states. Then, using $\not P_{-} \not P_{+}=2 b-\not P_{+} \not P_{-} \rightarrow 2 b-M m$, and the value of $a$ from Eq. (4.10), we get

$$
[\cdots]=-3
$$

and Eq. (C9) reduces to

$$
\mathcal{P}_{1 / 2}^{\mu \alpha}\left(P_{+}\right) D_{\alpha \beta} P_{1 / 2}^{\beta \nu}=-\frac{1}{3} \widetilde{\gamma}^{\mu}\left(P_{+}\right) \widetilde{\gamma}^{\nu}\left(P_{-}\right) .
$$

Similarly, using the same procedure we can show that

$$
\widetilde{g}^{\mu \alpha} D_{\alpha \beta} \mathcal{P}_{1 / 2}^{\beta \nu}=-\frac{1}{3} \widetilde{\gamma}^{\mu}\left(P_{+}\right) \widetilde{\gamma}^{\nu}\left(P_{-}\right) .
$$

Combining these results gives the result we seek

$$
\begin{aligned}
\mathcal{P}_{3 / 2}^{\mu \alpha}\left(P_{+}\right) D_{\alpha \beta} \mathcal{P}_{1 / 2}^{\beta \nu}= & \widetilde{g}^{\mu \alpha} D_{\alpha \beta} \mathcal{P}_{1 / 2}^{\beta \nu} \\
& -\mathcal{P}_{1 / 2}^{\mu \alpha}\left(P_{+}\right) D_{\alpha \beta} \mathcal{P}_{1 / 2}^{\beta \nu}=0 .
\end{aligned}
$$

\section{APPENDIX D: AN EXAMPLE OF THE IMPORTANCE OF THE COLLINEARITY CONDITION}

To give some insight into the importance of the collinear frame in the definitions of fixed-axis polarization states, consider a simple example where the initial and final baryon are identical (both have mass $m$ ) and the four-momenta are not collinear

$$
P_{ \pm}^{\prime}=\left(E^{\prime}, p \sin \theta, 0, \pm p \cos \theta\right)
$$

with $E^{\prime}=\sqrt{m^{2}+p^{2}}$. We can transform these momenta to a collinear frame by boosting in the $x$ direction using the transformation

$$
B_{x}=\left[\begin{array}{cccc}
\cosh \eta & \sinh \eta & 0 & 0 \\
\sinh \eta & \cosh \eta & 0 & 0 \\
0 & 0 & 1 & 0 \\
0 & 0 & 0 & 1
\end{array}\right] .
$$

Collinearity is achieved if

$$
\sinh \eta E^{\prime}+\cosh \eta p \sin \theta=0,
$$

and the resulting collinear four momenta are

$$
P_{ \pm}=B_{x} P_{ \pm}^{\prime}=(E, 0,0, \pm p \cos \theta)
$$

with $E=\sqrt{m^{2}+p^{2} \cos ^{2} \theta}$.

In this example, consider the longitudinal polarization vectors only. In the collinear frame they are

$$
\varepsilon_{0 P_{ \pm}}=\frac{1}{m}( \pm p \cos \theta, 0,0, E) .
$$

Their scalar product is

$$
m^{2} \varepsilon_{0 P_{+}} \cdot \varepsilon_{0 P_{-}}=-p^{2} \cos ^{2} \theta-E^{2}=-P_{+} \cdot P_{-} .
$$

In the original, non-collinear frame, the longitudinal polarizations are

$$
\begin{aligned}
\varepsilon_{0 P_{ \pm}^{\prime}} & =B_{x}^{-1} \varepsilon_{0 P_{ \pm}} \\
& =\frac{1}{m}( \pm \cosh \eta p \cos \theta, \mp \sinh \eta p \cos \theta, 0, E) .
\end{aligned}
$$

Hence,

$$
\begin{aligned}
m^{2} \varepsilon_{0 P_{+}^{\prime}} \cdot \varepsilon_{0 P_{-}^{\prime}} & =-\left(p^{2} \cos ^{2} \theta+E^{2}\right) \\
& =-\left(2 p^{2} \cos ^{2} \theta+m^{2}\right)=-P_{+}^{\prime} \cdot P_{-}^{\prime}
\end{aligned}
$$

showing that the scalar product of the two longitudinal polarization vectors $\varepsilon_{0 P_{+}} \cdot \varepsilon_{0 P_{-}}$is invariant and uniquely defined.

Now, suppose we were to define the longitudinal vectors in the original frame. One way to do this would be to observe that the two momenta (D1) can be obtained from the vectors

$$
\widetilde{P}_{ \pm}=\left(E^{\prime}, 0,0, \pm p\right)
$$


by rotation about the $y$ axis by an angle $\pm \theta$

$$
R_{ \pm \theta}=\left[\begin{array}{cccc}
1 & 0 & 0 & 0 \\
0 & \cos \theta & 0 & \pm \sin \theta \\
0 & 0 & 1 & 0 \\
0 & \mp \sin \theta & 0 & \cos \theta
\end{array}\right]
$$

The longitudinal vectors corresponding to (D9) are

$$
\widetilde{\varepsilon}_{ \pm}=\frac{1}{m}\left( \pm p, 0,0, E^{\prime}\right)
$$

so the new polarization vectors would be

$$
\begin{aligned}
\widetilde{\varepsilon}_{ \pm}^{\prime} & =R_{ \pm \theta} \widetilde{\varepsilon}_{ \pm} \\
& =\frac{1}{m}\left( \pm p, \pm \sin \theta E^{\prime}, 0, \cos \theta E^{\prime}\right) .
\end{aligned}
$$

These vectors are completely different than the correct ones given in Eq. (D7), and in particular

$$
\begin{aligned}
m^{2} \widetilde{\varepsilon}_{+}^{\prime} \cdot \widetilde{\varepsilon}_{-}^{\prime} & =-\left(p^{2}-\sin ^{2} \theta E^{2}+\cos ^{2} \theta E^{\prime 2}\right) \\
& =-\left(2 p^{2} \cos ^{2} \theta+m^{2} \cos 2 \theta\right) \\
& \neq-P_{+}^{\prime} \cdot P_{-}^{\prime}
\end{aligned}
$$

In conclusion: the correct way to treat fixed-axis polarization vectors is to transform to a collinear frame (if necessary), define the fixed-axis vectors there, and then do the inverse transformation back to the original frame (if desired).

\section{APPENDIX E: $D^{\mu \nu}$ IN THE COLLINEAR FRAME}

Consider the tensor

$$
D^{\mu \nu}=\sum_{\lambda} \varepsilon_{\lambda P_{+}}^{\mu} \varepsilon_{\lambda P_{-}}^{\nu *}
$$

where both $\varepsilon_{\lambda P_{+}}$are polarization vectors initially oriented along the $\hat{z}$ axis (in a collinear frame) and satisfy the constraints $P_{+} \cdot \varepsilon_{P_{+}}=P_{-} \cdot \varepsilon_{P_{-}}=0$ with

$$
P_{ \pm}=\left(E_{ \pm}, 0,0, p_{ \pm}\right)
$$

and $P_{+}^{2}=M^{2}, P_{-}^{2}=m^{2}$. $D^{\mu \nu}$ is a sum of direct products of four-vectors, and therefore is a covariant tensor. The explicit form of the $\varepsilon_{\lambda P_{ \pm}}$is

$$
\begin{aligned}
& \varepsilon_{\lambda P_{ \pm}}=\frac{1}{\sqrt{2}}(0,-\lambda,-i, 0) \text { if } \lambda= \pm \\
& \varepsilon_{0 P_{-}}=\frac{1}{m}\left(p_{-}, 0,0, E_{-}\right) \\
& \varepsilon_{0 P_{+}}=\frac{1}{M}\left(p_{+}, 0,0, E_{+}\right) .
\end{aligned}
$$

Using these explicit forms and interpreting $\varepsilon_{P_{+}}^{\mu}$ as a column vector and $\varepsilon_{P_{-}}^{\nu *}$ as a row vector, we get the following matrix form for $D$

$$
D^{\mu \nu}=\frac{1}{M m}\left[\begin{array}{cccc}
p_{+} p_{-} & 0 & 0 & p_{+} E_{-} \\
0 & M m & 0 & 0 \\
0 & 0 & M m & 0 \\
E_{+} p_{-} & 0 & 0 & E_{+} E_{-}
\end{array}\right] .
$$

The covariant form for this tensor can be found by exploiting the fact that $P_{+\mu} D^{\mu \nu}=0$ and $D^{\mu \nu} P_{-\nu}=0$. Hence the most general form of $D^{\mu \nu}$ is

$$
\begin{aligned}
D^{\mu \nu} & =a_{1}\left(-g^{\mu \nu}+\frac{P_{-}^{\mu} P_{+}^{\nu}}{b}\right)+ \\
& +a_{2}\left(P_{-}-\frac{b P_{+}}{M^{2}}\right)^{\mu}\left(P_{+}-\frac{b P_{-}}{m^{2}}\right)^{\nu}
\end{aligned}
$$

where $b=P_{+} \cdot P_{-}$and $a_{1}=1$ (to give the correct $D^{x x}$ and $D^{y y}$ components). The coefficient $a_{2}$ can be found from the trace

$$
D_{\mu}^{\mu}=-2-\frac{P_{+} \cdot P_{-}}{M m}
$$

which gives

$$
a_{2}=-\frac{M m}{b(M m+b)} .
$$

It is easy to verify that the two forms (E4) and (E5) are identical.

\section{APPENDIX F: THE CURRENT $J^{\mu}$ FOR THE $\gamma \mathbf{N} \rightarrow \Delta$ TRANSITION}

Equations (5.18) and (5.19) can be written

$$
\begin{aligned}
& J_{\Delta N}^{\mu}\left(P_{+}, P_{-}\right) \\
& \quad=-\frac{1}{\sqrt{3}} f_{v} \bar{w}_{\beta}\left(P_{+}, \lambda_{+}\right)\left[\gamma^{\mu} D^{\beta \alpha} \gamma_{\alpha} \gamma^{5}\right] u\left(P_{-}, \lambda_{-}\right) \mathcal{I},
\end{aligned}
$$

where the form factor $f_{v}$ was defined in Eq. (5.2) and

$$
\mathcal{I}=\int_{k} \psi_{\Delta}\left(P_{+}, k\right) \psi_{N}\left(P_{-}, k\right) .
$$

The operator in the square brackets in Eq. (F1) is reduced using the form of $D^{\beta \alpha}$ given in Eq. (4.9), remembering that the properties of the Rarita-Schwinger wave function imply that terms proportional to $P_{+}^{\beta}$ and $\gamma^{\beta}$ (when operating to the left) are zero, and that the Dirac equation may be used to replace $\mathbb{P}_{-} \rightarrow m$ when operating to the right, and $\not P_{+} \rightarrow M$ when operating to the left. We get

$$
\begin{aligned}
\gamma^{\mu} D^{\beta \alpha} \gamma_{\alpha} \gamma^{5} & =\gamma^{\mu}\left[-\gamma^{\beta}-A q^{\beta}\left(\not P_{+}-M\right)\right] \gamma^{5} \\
& =\left[2 A\left(M \gamma^{\mu}-P_{+}^{\mu}\right) q^{\beta}-2 g^{\mu \beta}\right] \gamma^{5}
\end{aligned}
$$

where

$$
A=\frac{1}{M m+b}=\frac{2}{(M+m)^{2}+Q^{2}} .
$$

Noting that $P_{+}^{\mu}=P^{\mu}+\frac{1}{2} q^{\mu}$, the operator (F3) can be written in terms of the invariants of (5.11)

$$
\begin{aligned}
& \gamma^{\mu} D^{\beta \alpha} \gamma_{\alpha} \gamma^{5} \\
& \quad=\left[g_{1} q^{\beta} \gamma^{\mu}+g_{2} q^{\beta} P^{\mu}+g_{3} q^{\beta} q^{\mu}-g_{4} g^{\mu \nu}\right] \gamma^{5}
\end{aligned}
$$


where

$$
\begin{aligned}
& g_{1}=2 M A \\
& g_{2}=-2 A \\
& g_{3}=-A \\
& g_{4}=2 .
\end{aligned}
$$

Note that

$$
g_{4}=(M+m) g_{1}+\frac{M^{2}-m^{2}}{2} g_{2}-Q^{2} g_{3}
$$

as required by current conservation, Eq. (5.13). The Jones and Scadron form factors are

$$
G_{i}=f_{v} g_{i} \frac{\mathcal{I}}{\sqrt{3}} .
$$

We conclude that the physical form factors are, within the $S$-wave model,

$$
\begin{aligned}
& G_{M}^{*}\left(Q^{2}\right)=\frac{8 m}{3 \sqrt{3}(M+m)} f_{v} \mathcal{I} \\
& G_{E}^{*}\left(Q^{2}\right)=G_{C}^{*}\left(Q^{2}\right)=0 .
\end{aligned}
$$

\section{APPENDIX G: ASYMPTOTIC $Q^{2}$ DEPENDENCE OF THE INVARIANT BODY INTEGRALS}

In this appendix we discuss the asymptotic dependence of the "body" integrals

$$
B_{H}\left(Q^{2}\right)=\int_{k} \psi_{H}\left(P_{+}, k\right) \psi_{N}\left(P_{-}, k\right),
$$

where $H=N$ or $\Delta$. [5]. The high $Q^{2}$ dependence of $B_{H}\left(Q^{2}\right)$ determines the asymptotic behavior of the nucleon and $N \rightarrow \Delta$ form factors. To simplify the discussion we consider the easiest case, when the parameters of wave function are $\beta_{1}=\beta_{2}=\beta$ for the nucleon, $\alpha_{1}=\alpha_{2}=\alpha$ for the $\Delta$, and we will sometimes use the notation $\beta_{H}$, where $\beta_{N}=\beta$ and $\beta_{\Delta}=\alpha$.

The integral $B$ is covariant and may be evaluated in any frame. It is convenient to evaluate it in the "antilab" frame, where the final hadron is at rest. In this case the momenta are $P_{H}=\left(m_{H}, 0,0,0\right)$ and $P_{q_{0}}=$ $\left(E_{0}, 0,0,-q_{0}\right)$, with $E_{0}=\sqrt{m^{2}+q_{0}^{2}}$ the nucleon energy in the initial state. The photon four-momentum is then $q=\left(m_{H}-E_{0}, 0,0, q_{0}\right)$ with

$$
q_{0}^{2}=\left(\frac{Q^{2}+m_{H}^{2}+m^{2}}{2 m_{H}}\right)^{2}-m^{2} \rightarrow \frac{Q^{4}}{4 m_{H}^{2}}
$$

as $Q^{2} \rightarrow \infty$. With these momenta we can write the body integral as

$$
B_{H}\left(Q^{2}\right)=\frac{N_{0}}{(2 \pi)^{2}} \int_{0}^{\infty} \frac{k^{2} d k}{2 m_{s} E_{s}} \psi_{H}\left(P_{H}, k\right) I\left(Q^{2}\right),
$$

where

$$
I\left(Q^{2}\right)=\int_{-1}^{1} \frac{d z}{\left(\beta-2+2 \frac{E_{0}}{m} \frac{E_{s}}{m_{s}}+2 \frac{q_{0}}{m} \frac{k}{m_{s}} z\right)^{2}} .
$$

In this frame only the initial state depends of the angular coordinate $z=\cos \theta$. Introducing the parameter

$$
\eta=\frac{(\beta-2) m m_{s}+2 E_{0} E_{s}}{2 q_{0} k}
$$

gives

$$
\begin{aligned}
I\left(Q^{2}\right) & =\frac{m^{2} m_{s}^{2}}{4 q_{0}^{2} k^{2}} \int_{-1}^{1} \frac{d z}{(z+\eta)^{2}}=\frac{m^{2} m_{s}^{2}}{2 q_{0}^{2} k^{2}\left(\eta^{2}-1\right)} \\
& \rightarrow \frac{m^{2}}{2 q_{0}^{2}}
\end{aligned}
$$

as $Q^{2} \rightarrow \infty$. Motivated by the nonrelativistic definition of the wave function at the origin, we define the following covariant integral

$$
\widetilde{\psi}_{H}(0) \equiv \int_{0}^{\infty} \frac{k^{2} d k}{2 m_{s} E_{s}} \psi_{H}\left(P_{H}, k\right) .
$$

Our results can now be expressed in terms of the behavior of this integral.

Case I: If the integral (G6) exists, then the diquark momentum $k$ is localized, and the limit (G5) can be taken under the integral, leading to the result

$$
\begin{aligned}
\lim _{Q^{2} \rightarrow \infty} B_{H}\left(Q^{2}\right) & =\frac{N_{0}}{(2 \pi)^{2}} \widetilde{\psi}_{H}(0) I\left(Q^{2}\right) \\
& \rightarrow \frac{N_{0}}{(2 \pi)^{2}} \widetilde{\psi}_{H}(0) \frac{2 m_{H}^{2} m^{2}}{Q^{4}} .
\end{aligned}
$$

We obtain the interesting (and well known) result that, if cases where the value of the relativistic wave function of one of the hadrons is finite at the origin, the asymptotic from factor is determined by the high momentum behavior of the other wave function. For the models used in this paper this shows that the $N \rightarrow \Delta$ body form factors go like $Q^{-4}$ are large $Q^{2}$.

Case II: If $\widetilde{\psi}(0)$ does not exist, the limit (G5) cannot be taken and the analysis of the large $Q^{2}$ behavior depends on the behavior of the full integral. In this case we return to (G3) and write $(H=N$ now)

$$
B_{N}\left(Q^{2}\right)=\frac{N_{0}^{2}}{(8 \pi)^{2}} \mathcal{I}\left(Q^{2}\right)
$$

The integral $\mathcal{I}$ can be evaluated in the diquark energy scaled by the diquark mass $x=E_{s} / m_{s}$ considering $k=$ $m_{s} \sqrt{x^{2}-1}$. As result we have for $H=N$

$$
\mathcal{I}\left(Q^{2}\right)=\int_{1}^{\infty} \frac{g(x)}{D(x+\omega)^{2}} d x,
$$


where $g(x)=\sqrt{x^{2}-1} ; \omega=\frac{1}{2}(\beta-2)$ and the denominator $D$ is

$$
\begin{aligned}
D & =x^{2}+2 \omega \frac{E_{0}}{m} x+\frac{q_{0}^{2}}{m^{2}}+\omega^{2} \\
& \rightarrow x^{2}+2 \omega \frac{q_{0}}{m} x+\frac{q_{0}^{2}}{m^{2}} .
\end{aligned}
$$

The last approximation holds at large $q_{0}$, where all constant terms and terms proportional to $x / q_{0}$ can be neglected, because they are always smaller than terms proportional to $x^{2},\left(q_{0} / m\right)^{2}$, or $x q_{0} / m$. Also for large $q_{0}$ we can re-write (G9) as

$$
\begin{aligned}
\mathcal{I}\left(Q^{2}\right) & \simeq \frac{m^{2}}{q_{0}^{2}} \int_{1}^{\infty} g(x)\left\{\frac{1}{(x+\omega)^{2}}-\frac{1}{x^{2}+2 \omega \frac{q_{0}}{m} x+\frac{q_{0}^{2}}{m^{2}}}\right\} d x \\
& -2 \omega \frac{m^{3}}{q_{0}^{3}} \int_{1}^{\infty} g(x)\left\{\frac{1}{x+\omega}-\frac{x+2 \omega \frac{q_{0}}{m}-\omega}{x^{2}+2 \omega \frac{q_{0}}{m} x+\frac{q_{0}^{2}}{m^{2}}}\right\} d x .
\end{aligned}
$$

Note that each term inside the brackets diverges but the result is convergent. The above integrals can be performed analytically following the usual techniques. Considering $x=1 / \cos u$ the integrand function becomes an algebraic function of $\cos u$ and $\sin u$ that we integrate analytically using the Mathematica program. Considering only the leading and next leading terms in $q_{0} / \mathrm{m}$ in the the general expressions, one is left with

$$
\mathcal{I} \rightarrow \frac{m^{2}}{q_{0}^{2}}\left[\log \left(\frac{2 q_{0}}{m}\right)-\mathcal{R}(\omega)\right]
$$

where

$$
\begin{aligned}
& \mathcal{R}(\omega)=1+\frac{\omega}{\sqrt{1-\omega^{2}}} \times \\
& {\left[2 \tan ^{-1}\left(\frac{1-\omega}{\sqrt{1-\omega^{2}}}\right)+\tan ^{-1}\left(\frac{\sqrt{1-\omega^{2}}}{\omega}\right)\right] .}
\end{aligned}
$$

The analytical continuation of the (G12) for the case $\omega \geq 1$ (or $\beta \geq 2$ ) is obtained considering the relation between logarithms and arc-tangent $\log \frac{1+i x}{1-i x}=2 i \tan ^{-1}(x)$ and the replacement $\sqrt{1-\omega^{2}} \equiv-i \sqrt{\omega^{2}-1}$.

In conclusion, we can write

$$
B_{N}\left(Q^{2}\right) \rightarrow \frac{N_{0}^{2}}{(4 \pi)^{2}} \frac{m^{4}}{Q^{4}} \log \frac{Q^{2}}{m^{2}} .
$$

This term is independent of $\beta$. The nonleading terms in $Q^{-4}$ carry the dependence in the parameter $\beta$ and set the scale of the logarithm behavior.

This logarithmic dependence of the nucleon form factors was missed in Ref. [29], but this oversight does not affect any of the conclusions of that paper.
[1] M. K. Jones et al. [Jefferson Lab Hall A Collaboration], Phys. Rev. Lett. 84, 1398 (2000).

[2] O. Gayou et al. [Jefferson Lab Hall A Collaboration], Phys. Rev. Lett. 88, 092301 (2002).

[3] V. Punjabi et al., Phys. Rev. C 71, 055202 $\begin{array}{lllll}\text { (2005) [Erratum-ibid. C 71, } 069902 & \text { (2005)] }\end{array}$ arXiv:nucl-ex/0501018.

[4] A. Kvinikhidze and G. A. Miller, Phys. Rev. C 73, 065203 (2006) arXiv:nucl-th/0603035.

[5] F. Gross, G. Ramalho and M. T. Peña, Phys. Rev. C 77, 015202 (2008).

[6] C. E. Hyde-Wright and K. de Jager, Ann. Rev. Nucl. Part. Sci. 54, 217 (2004) arXiv:nucl-ex/0507001.

[7] J. Arrington, C. D. Roberts and J. M. Zanotti, arXiv:nucl-th/0611050.

[8] R. Beck et al., Phys. Rev. C 61, 035204 (2000) arXiv:nucl-ex/9908017]; T. Pospischil et al., Phys. Rev. Lett. 86, 2959 (2001) arXiv:nucl-ex/0010020; D. Elsner et al., Eur. Phys. J. A 27, 91 (2006) arXiv:nucl-ex/0507014; S. Stave et al., arXiv:nucl-ex/0604013

[9] G. Blanpied et al., Phys. Rev. C 64, 025203 (2001); G. Blanpied et al., Phys. Rev. Lett. 79, 4337 (1997).

[10] C. Mertz et al., Phys. Rev. Lett. 86, 2963 (2001) arXiv:nucl-ex/9902012]; N. F. Sparveris et al. [OOPS Collaboration], Phys. Rev. Lett. 94, 022003 (2005) arXiv:nucl-ex/0408003.

[11] V. V. Frolov et al., Phys. Rev. Lett. 82, 45 (1999) arXiv:hep-ex/9808024]. K. Joo et al. [CLAS Collaboration], Phys. Rev. Lett. 88, 122001 (2002) arXiv:hep-ex/0110007.
[12] M. Ungaro et al. [CLAS Collaboration], Phys. Rev. Lett. 97, 112003 (2006) arXiv:hep-ex/0606042.

[13] V. Pascalutsa, M. Vanderhaeghen and S. N. Yang, Phys. Rept. 437, 125 (2007) arXiv:hep-ph/0609004.

[14] F. Gross, G. Ramalho and M. T. Peña, arXiv:0708.0995 [nucl-th]. To appear in Phys. Rev. C.

[15] F. Gross, Phys. Rev. 186, 1448 (1969).

[16] F. Gross, Phys. Rev. C 26, 2226 (1982).

[17] F. Gross, J. W. Van Orden and K. Holinde, Phys. Rev. C 45, 2094 (1992).

[18] A. Stadler and F. Gross, Phys. Rev. Lett. 78, 26 (1997).

[19] A. Stadler, F. Gross, and M. Frank, Phys. Rev. C 56, 2396 (1997).

[20] F. Gross, A. Stadler and M. T. Peña, Phys. Rev. C 69, 034007 (2004).

[21] J. Adam, F. Gross, C. Savkli and J. W. Van Orden, function," Phys. Rev. C 56, 641 (1997).

[22] F. Gross and D. O. Riska, Phys. Rev. C 36, 1928 (1987).

[23] C. Savkli and F. Gross, Phys. Rev. C 63, 035208 (2001) arXiv:hep-ph/9911319.

[24] W. Rarita and J. S. Schwinger, Phys. Rev. 60, 61 (1941).

[25] F. J. Milford Phys. Rev. 98, 1488 (1955).

[26] M. Benmerrouche, R. M. Davidson and N. C. Mukhopadhyay, Phys. Rev. C 39 (1989) 2339.

[27] H. Haberzettl, arXiv:nucl-th/9812043

[28] G. Ramalho, M. T. Peña, and F. Gross, in preparation.

[29] F. Gross and P. Agbakpe, Phys. Rev. C 73, 015203 (2006).

[30] A. Kvinikhidze and G. A. Miller, arXiv:nucl-th/0701017

[31] M. Jacob and G. C. Wick, Annals Phys. 7, 404 (1959) 
[Annals Phys. 281, 774 (2000)].

[32] C. E. Carlson, Phys. Rev. D 34, 2704 (1986); C. E. Carlson and N. C. Mukhopadhyay, Phys. Rev. Lett. 81, 2646 (1998) arXiv:hep-ph/9804356; C. E. Carlson, Few Body Syst. Suppl. 11, 10 (1999) arXiv:hep-ph/9809595.

[33] H. F. Jones and M. D. Scadron, Annals Phys. 81, 1 (1973).

[34] J. F. Donoghue, E. Golowich and B. R. Holstein, Phys. Rev. D 12, 2875 (1975).

[35] N. Isgur, G. Karl and R. Koniuk, Phys. Rev. D 25, 2394 (1982).

[36] M. Warns, W. Pfeil and H. Rollnik, Phys. Rev. D 42, 2215 (1990).

[37] S. Capstick and B. D. Keister, Phys. Rev. D 51, 3598 (1995) arXiv:nucl-th/9411016.

[38] R. Bijker, F. Iachello and A. Leviatan, Annals Phys. 236, 69 (1994) arXiv:nucl-th/9402012.

[39] B. Julia-Diaz, D. O. Riska and F. Coester, Phys. Rev. C 69, 035212 (2004) [Erratum-ibid. C 75, 069902 (2007)] arXiv:hep-ph/0312169.

[40] B. Julia-Diaz and D. O. Riska, Nucl. Phys. A 757, 441 (2005) arXiv:nucl-th/0411012.

[41] B. Julia-Diaz, T. S. Lee, T. Sato and L. C. Smith, Phys. Rev. C 75, 015205 (2007).

[42] V. M. Braun, A. Lenz, G. Peters and A. V. Radyushkin, Phys. Rev. D 73, 034020 (2006) arXiv:hep-ph/0510237.

[43] P. Stoler, Phys. Rev. D 65, 053013 (2002) arXiv:hep-ph/0108257; P. Stoler, Phys. Rev. Lett. 91, 172303 (2003) arXiv:hep-ph/0210184.

[44] M. Guidal, M. V. Polyakov, A. V. Radyushkin and M. Vanderhaeghen, Phys. Rev. D 72, 054013 (2005) arXiv:hep-ph/0410251.

[45] V. Pascalutsa, C. E. Carlson and M. Vanderhaeghen, Phys. Rev. Lett. 96, 012301 (2006) arXiv:hep-ph/0509055.

[46] V. D. Burkert and T. S. H. Lee, Int. J. Mod. Phys. E 13,
1035 (2004).

[47] D. Drechsel and L. Tiator, AIP Conf. Proc. 904, 129 (2007) arXiv:nucl-th/0610112.

[48] T. Sato and T. S. H. Lee, Phys. Rev. C 54, 2660 (1996) arXiv:nucl-th/9606009; T. Sato and T. S. H. Lee, Phys. Rev. C 63, 055201 (2001) arXiv:nucl-th/0010025.

[49] S. S. Kamalov, S. N. Yang, D. Drechsel, O. Hanstein and L. Tiator, Phys. Rev. C 64, 032201 (2001) arXiv:nucl-th/0006068; S. S. Kamalov and S. N. Yang, Phys. Rev. Lett. 83, 4494 (1999) arXiv:nucl-th/9904072.

[50] G. L. Caia, V. Pascalutsa, J. A. Tjon and L. E. Wright, Phys. Rev. C 70, 032201 (2004) arXiv:nucl-th/0407069; V. Pascalutsa and J. A. Tjon, Phys. Rev. C 70, 035209 (2004) arXiv:nucl-th/0407068.

[51] V. D. Burkert, Prog. Part. Nucl. Phys. 55 (2005) 108.

[52] B. Julia-Diaz, private comunication (2007).

[53] W. Bartel et al., Phys. Lett. B 28, 148 (1968).

[54] S. Stein et al., Phys. Rev. D 12, 1884 (1975).

[55] V. Pascalutsa and M. Vanderhaeghen, Phys. Rev. Lett. 95, 232001 (2005) arXiv:hep-ph/0508060.

[56] V. Pascalutsa and M. Vanderhaeghen, Phys. Rev. D 73, 034003 (2006) arXiv:hep-ph/0512244.

[57] A. Faessler, T. Gutsche, B. R. Holstein, V. E. Lyubovitskij, D. Nicmorus and K. Pumsa-ard, arXiv:hep-ph/0612246

[58] C. Fernandez-Ramirez, E. M. de Guerra and J. M. Udias, Eur. Phys. J. A 31, 572 (2007) arXiv:nucl-th/0611062.

[59] T. A. Gail and T. R. Hemmert, Eur. Phys. J. A 28, 91 (2006) arXiv:nucl-th/0512082.

[60] C. Alexandrou, Ph. de Forcrand, H. Neff, J. W. Negele, W. Schroers and A. Tsapalis, Phys. Rev. Lett. 94, 021601 (2005) arXiv:hep-lat/0409122.

[61] C. Alexandrou, G. Koutsou, H. Neff, J. W. Negele, W. Schroers and A. Tsapalis, arXiv:0710.4621 [hep-lat]. 